\title{
Model-based control of vortex shedding at low Reynolds numbers
}

\author{
Simon J. Illingworth
}

Received: date / Accepted: date

\begin{abstract}
Model-based feedback control of vortex shedding at low Reynolds numbers is considered. The feedback signal is provided by velocity measurements in the wake, and actuation is achieved using blowing and suction on the cylinder's surface. Using two-dimensional direct numerical simulations and reduced-order modelling techniques, linear models of the wake are formed at Reynolds numbers between 45 and 110 . These models are used to design feedback controllers using $\mathcal{H}_{\infty}$ loopshaping. Complete suppression of shedding is demonstrated up to $\mathrm{Re}=110$-both for a single-sensor arrangement and for a three-sensor arrangement. The robustness of the feedback controllers is also investigated by applying them over a range of off-design Reynolds numbers, and good robustness properties are seen. It is also observed that it becomes increasingly difficult to achieve acceptable control performance - measured in a suitable way - as Reynolds number increases.
\end{abstract}

Keywords flow control $\cdot$ fluid mechanics $\cdot$ reduced-order model $\cdot$ vortex shedding

Acknowledgements The author would like to thank Hiroshi Naito and Koji Fukagata for helpful discussions concerning the direct numerical simulations. The author gratefully acknowledges financial support from the Japan Society for the Promotion of Science (JSPS), who supported him as a Research Fellow during part of this work.

\section{Introduction}

The von Kármán vortex street in the two-dimensional cylinder wake first appears at a Reynolds number near 49 [47]. This vortex street gives rise to increased drag and unsteady lift forces. The advantages of suppressing these vortices is therefore clear, and one way to achieve this is using active flow control. Active flow control schemes employ actuators to provide external energy to a fluid flow, thereby controlling it in some desired way. This actuation may be predetermined

Simon J. Illingworth

Department of Mechanical Engineering, University of Melbourne, VIC 3010, Australia

E-mail: sillingworth@unimelb.edu.au 
(open-loop), or may respond to some suitably-chosen sensor measurement via some feedback law (closed-loop).

\subsection{Literature review}

There have been a number of studies of closed-loop control of vortex shedding. The first study was performed by Berger [3], which predates all other studies by more than twenty years. In his experiments an oval-shaped piezoelectric transducer served simultaneously as the cylinder and the actuator-the body moving transverse to the flow in response to an applied voltage - and a hot-wire probe served as the sensor. Vortex shedding, which occurred naturally for the oval-shaped cylinder at Reynolds numbers of 77 and above, was suppressed by feedback at Reynolds numbers up to 80 .

The study of Berger [3] shares a number of features with its earliest successors $[8,36,33,42]$ : all used a velocity measurement in the wake as the feedback signal; in all cases the control law was a simple proportional feedback gain chosen by trial and error (possibly with some phase shift); and all were conducted at low Reynolds numbers near the onset of vortex shedding. (A notable exception is the study by Ffowcs Williams \& Zhou [8], performed at a Reynolds number of 400.) The actuation strategies employed in these early studies included transverse displacement of the cylinder [3,42], loudspeakers $[8,36]$ and blowing and suction on the cylinder surface [33].

All of these early studies successfully eliminated vortex shedding, but did so only slightly above the critical Reynolds number. Roussopoulos [36], for example, increased the critical Reynolds number by about $20 \%$ in his experimental study, whilst Park et al. [33] increased it by about $27 \%$ in simulations. Gunzburger \& Lee [15] achieved complete suppression at slightly higher Reynolds numbers (of $\operatorname{Re}=80$, about $60 \%$ above criticality) by using surface pressure measurements as the feedback signal and by adding an additional orifice for blowing and suction at the cylinder's trailing edge. Keles [23] used a similar experimental arrangement to Roussopoulos [36] at a higher Reynolds number of 170. The amplitude of vortex shedding was reduced along the entire span of the wake at a streamwise location two cylinder diameters downstream, but it is not clear whether a similar reduction occurred for all streamwise positions. Cohen et al. [7] also used a proportional feedback gain but, rather than use any sensor measurement directly, the controller acted on the first POD mode of a reduced-order model. The amplitude of the POD mode was estimated from several sensor measurements using linear stochastic estimation. Siegel et al. [38] extended this work by applying proportional-differential control to the first POD mode. Recently, Illingworth et al. [20] have demonstrated the severe limitations of proportional feedback control for vortex shedding.

The first break from simple trial-and-error-based control laws can be attributed to Gillies [12], who used multiple sensors, a POD-based reduced-order model, and a neural network-based control algorithm. Graham et al. [14] applied optimal control theory to a POD-based model of the cylinder wake at $\mathrm{Re}=100$, while Min \& Choi [31] applied three different suboptimal control schemes at Reynolds numbers up to 160. These more systematic studies were followed by a number of optimal controlbased studies using either the Navier-Stokes equations directly $[18,35,27,17]$ or a POD-based reduced-order model $[39,5,4]$. 
Instead of designing a control law based on the Navier-Stokes equations directlyor a reduced-order representation of them - an alternative approach has been to base the control law on Föppl's point vortex model. By doing so and then applying the control law in direct numerical simulations of a cylinder, Li \& Aubry [26] were able to maintain the lift force close to zero at Reynolds numbers of 100 and 200. The feedback signal was the unsteady lift force, and actuation was achieved by transverse displacement of the cylinder. Using a similar approachthis time measuring the centreline velocity in the wake and rotating the cylinder to actuate - Protas [34] stabilized the downstream region of the wake in DNS at Re $=75$ using a Linear Quadratic Gaussian (LQG) regulator, but oscillations were actually made worse in the near-wake region.

A recent approach has been to again use a simple proportional gain feedback law, but to be more systematic in its choice. Weller et al. [45] used 16 sensors in the wake of a square cylinder to suppress shedding at $R e=150$ using blowing and suction on its surface. A proportional gain for each sensor was chosen in real time using a gradient descent method. In related work, Camarri \& Iollo [6] used linear stability and sensitivity analyses to choose the sensor location and proportional feedback gain for a square cylinder at Reynolds numbers up to 140. The single-sensor and two-sensor cases were considered. In still more recent work, Son et al. [41] investigated vortex shedding control using linear proportional, proportional-differential and proportional-integral control at Reynolds numbers of 60 and 100. Good results are seen but, with no model to inform control design, this large parameter space was investigated by trial and error.

\section{$1.2 *$ This study}

This paper investigates model-based feedback control of vortex shedding at low Reynolds numbers. The control objective is to completely eliminate vortex shedding and render the flow steady. Like a number of previous studies, actuation is provided by blowing and suction at the cylinder surface, and sensing is provided by a velocity measurement in the wake. The controllers are model-based, meaning that a model of the system is used in the control design process. The controllers are also robust, synthesized using methods from robust control theory. This means that the controllers should still perform well in the presence of modelling uncertainty (i.e. a mismatch between the model used for control and the real system) and at off-design operating conditions.

Model-based feedback control has certainly been applied in fluid mechanics before - see [24] for a review, and [2] for an overview of their application to the Ginzburg-Landau system. A novel aspect of this paper is the way in which it combines system identification techniques (or, put another way, data-based model reduction) with robust control methods (specifically $\mathcal{H}_{\infty}$ loop shaping) to control vortex shedding. Rather than use the POD to generate the data-based models - as has been done in a number of previous studies - system identification techniques (specifically the Eigensystem Realization Algorithm) are used instead. This choice is motivated by the fact that the POD is optimal in capturing the energy in a flow, but not necessarily its input-output behaviour, which is what is important for feedback control purposes [37]. In contrast, the Eigensystem Realization Algorithm produces reduced-order models that are balanced (referring to the fact 
that their observability and controllability gramians are equal and diagonal), and therefore correctly captures the system's input-output behaviour. As well as designing controllers that are robust, their robustness is actually tested by applying them over a wide range of off-design Reynolds numbers.

The paper also considers the control performance achieved as Reynolds number increases. As already mentioned, it has been observed in a number of the early, trial-and-error-based studies that vortex shedding could be completely eliminated at Reynolds number slightly above critical, but not much higher [3,36,33]. One can therefore say that these early investigators met with an increasingly challenging control problem as Reynolds number increased. However, as shown in [20], this is due to the simple control laws that these early studies used, and is overcome by the model-based control methods employed in this paper and others. In a separate study, [25] considered model-based feedback control of the linearized GinzburgLandau equation using a single sensor in the wake. They employed model-based optimal control over a range of Reynolds numbers. (To be more precise, a bifurcation parameter is varied, which serves as a surrogate for the the Reynolds number in this simple system.) Despite employing model-based control, they observed that control became more challenging as the Reynolds number increased, and this was attributed to a gradual decay of stabilizability with increasing Reynolds number. In other words, control became increasingly challenging, but this was not due to any deficiencies in the control laws (as in earlier studies), but rather due to the nature of the plant itself. We will see in $\S 3$ that a similar reduction in control performance, as measured in a suitable way, applies to the cylinder wake over the range of Reynolds numbers considered.

The paper is organized as follows. $\S 2$ describes the numerical scheme; control arrangement and control design; and reduced-order modelling techniques to be used. $\S 3$ presents results of feedback control in DNS for Reynolds numbers between 45 and 110. As well as investigating the performance and robustness of the controllers, it is also seen that control becomes more challenging as the Reynolds number increases. Conclusions are given in $\S 4$.

\section{Modelling and control of the cylinder wake}

\subsection{Numerical scheme}

The flow is solved using direct numerical simulation. The incompressible NavierStokes equations are non-dimensionalized using the fluid density, $\rho$, the free-stream velocity, $U_{\infty}$, and the cylinder diameter, $D$. The Reynolds number is defined by $R e=\rho U_{\infty} D / \mu$, where $\mu$ is the dynamic viscosity. The spatial discretization is performed in cylindrical coordinates using an energy-conservative finite-difference scheme $[10,32]$. Time integration is performed using a low-storage third-order Runge-Kutta/Crank-Nicolson scheme. The computational grid has 256 points in the circumferential direction; 220 points in the radial direction (clustered near the cylinder surface); and extends 78 cylinder radii from the cylinder's centre. Results have been validated using grid refinement and boundary placement studies, as well as comparison with experimental data. At Reynolds numbers between 60 and 100, the Strouhal numbers agree with the experimental parallel-shedding data of Williamson [46] to within $1 \%$. 


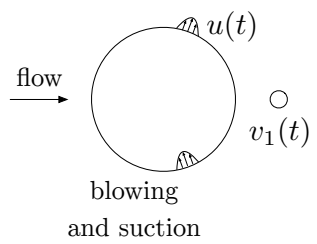

velocity sensors

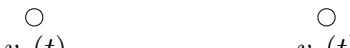

$v_{2}(t)$

$v_{3}(t)$

Fig. 1 Closed-loop control arrangement. Three velocity sensors are positioned at $0.76 D$, $2.75 \mathrm{D}$ and $4.78 \mathrm{D}$ from the cylinder's centre. The single-sensor arrangement uses only the second sensor $\left(v_{2}\right)$ at $2.75 \mathrm{D}$ for control. The three-sensor arrangement uses all sensors for control.

\subsection{Feedback control arrangement}

The feedback arrangement is shown in figure 1. Actuation is provided by antisymmetrical blowing and suction at the cylinder's surface. The centres of the two actuation regions are at angles of $\pm 70^{\circ}$ from the cylinder's downstream-pointing horizontal. This is the same as that used by Park et al. [33], who tested different angles and found this to be the optimum. This was attributed to the actuation lying just upstream of the separation angles that would occur in the unforced case. The distribution of each actuation region is Gaussian, specified such that the amplitude falls to $1 \%$ of its peak at an angle of $\pm 25^{\circ}$ from its centre. The actuation is normalized such that, for unity sensor measurement and unity feedback gain, the amplitude of the integral of the Gaussian distribution is also unity in each of the two regions.

Sensing is provided by velocity probes (which measure the transverse velocity) positioned downstream of the cylinder. There are three velocity probes positioned $0.76 D, 2.75 D$, and $4.78 D$ downstream of the cylinder's centre. These three sensors are used in two different configurations. The first is a single-sensor arrangement, which uses only the second probe at $2.75 D$. (This is the same arrangement as Park et al. [33].) The second arrangement uses all three velocity sensors.

\subsection{Finding a linear, reduced-order model}

In order to perform model-based control using $\mathcal{H}_{\infty}$ loop shaping (to be described in $\S 2.5$ ), we first require a linear model. Clearly, a linear model will not be able to explain the nonlinear, limit-cycling behaviour which will inevitably be observed in the uncontrolled wake. With this in mind, we justify using a linear model in two ways. First, the model will be valid for small perturbations about a steady base flow, and this is ultimately what we want to achieve with control. That is, it is sensible to linearize about where we want to be. Second, a linear control approach has been adopted in previous studies [33], and the present approach is therefore consistent with those earlier studies.

Having justified our using a linear model, let us now consider how such a model can be found. At the unstable Reynolds numbers considered the wake, left to its own devices, will develop into a limit-cycle, which is certainly not linear. To find a linear model, then, we must first stabilize the wake using feedback, because in doing so we also linearize it. Even though the wake is then operating in closed- 
loop, its open-loop dynamics can still be obtained - see Illingworth et al. [19] for more details on this approach.

It would seem that this approach to system identification requires that a stabilizing control law is already available. This is clearly problematic, since the primary purpose of the system identification is to find a model with which to design a controller. This is not the case, however, and we now consider why. Crucially, the cylinder wake at $\operatorname{Re}=45$ (the lowest Reynolds number considered) is stable. This means that a reduced-order model can be found without first stabilizing the wake, and therefore knowledge of a stabilizing control law is not required. Equally important, a controller designed for the $\mathrm{Re}=45$ wake (using its reduced-order model and $\mathcal{H}_{\infty}$ loop shaping) is sufficiently robust that, when applied at a higher, unstable Reynolds number, the resulting closed-loop system is stable. (This robustness of controllers will be considered in detail in §3.6.) To give a specific example for the three-sensor arrangement: a controller designed for $\mathrm{Re}=45$ is still stable when applied at Reynolds numbers of 50, 55, 60, 65 and 70. This means that a stabilizing control law at these unstable Reynolds numbers is now available, but that only a model at a stable Reynolds number was required to obtain it. This ultimately means that a controller found at one Reynolds number can serve as a stabilizing controller at another, thereby allowing a linear model at this second Reynolds number to be found. A controller designed for the $R e=70$ wake, for example, is stabilizing at all Reynolds numbers between 45 and 90, thereby allowing access to reduced-order models up to $\mathrm{Re}=90$. This 'leapfrogging' process can continue, providing access to higher Reynolds numbers at each iteration.

System identification in closed-loop is a well-established technique. To give some context, a review article on the method can be found as far back as Gustavsson et al. [16], and there have been a number of reviews since [43,9]. Perhaps the most compelling reason to use it is that, in experiments, system identification in closed-loop is the only way to generate linear models for a system which is open-loop unstable.

Now consider the linear, time-invariant dynamical system given in state-space form as

$$
\begin{aligned}
& \dot{x}(t)=A x(t)+B u(t) \\
& y(t)=C x(t)+D u(t),
\end{aligned}
$$

where $u \in \mathbb{R}^{p}$ is a vector of inputs, $y \in \mathbb{R}^{q}$ is a vector of outputs, $x \in \mathbb{R}^{n}$ is the system state of dimension $n$, and $A, B, C$ and $D$ are suitably-dimensioned matrices. The feedback arrangement used in this paper has a single input, $p=1$, and a single output, $q=1$.

In order to form a reduced-order model of the form (1) we use the Eigensystem Realization Algorithm (ERA). The starting point for the ERA is the system's impulse response, which is found in the cylinder's case directly from simulations. The magnitude of the impulse is 0.01 (see Appendix A). In order to verify that the wake responds linearly to this forcing, impulse response simulations have also been performed for amplitudes of 0.02, 0.05 and 0.1 . The wake responds linearly at all of these amplitudes, which span an order of magnitude, and we can therefore be confident that the dynamics remain linear for the magnitude of 0.01 used (which is the smallest of all those tested).

The ERA then proceeds by arranging the impulse response (which in this context is also referred to as the system's Markov parameters) in a particular 
way to form a block data matrix (a Hankel matrix). By factorizing this matrix using the singular value decomposition the matrices $A, B, C$ and $D$ in (1) are found. (Or rather their discrete-time equivalents - see Appendix A.) An important property of the ERA is that it produces models which are balanced, the balancing referring to the model's observability and controllability Gramians being equal and diagonal. Physically this means that the system's input-output behaviour is properly captured, and this is important for feedback control purposes. Further details on the ERA are available in Juang [21]. For details on its application to flow control, see Ma et al. [29], Illingworth et al. [19]. For a more detailed description of the ERA, see Appendix A, and for more details on the closed-loop system identification approach, see Appendix B.

\subsection{Model validation}

Before the reduced-order models are used for control design, they must first be validated. The model order chosen for all Reynolds numbers is $n=100$. This is sufficient to capture the dynamics of the wake, as will now be demonstrated. Before doing so, we first note that $n=100$ is a high model order and requires some explanation. This will be given in $\S 2.5$, since our choice of model order is closely related to feedback controller design.

Figure $2(a)$ (the first row) shows the open-loop impulse responses for the reduced-order models and compares them to direct numerical simulations at Reynolds numbers of 45, 60, 80 and 100. The agreement is excellent. Part (b) (the second and third rows) validates the models in the frequency domain (in a Bode plot) by comparing their frequency responses to those found using spectral analysis [28]. The spectral approach determines the frequency response directly by finding the complex-valued response at a discrete set of frequencies. This is achieved using MATLAB's spafdr command. This spectral approach may be likened to taking the ratio of the discrete Fourier transforms of the input and output signals, but with an important refinement: to form the frequency response at some frequency $\omega$, it uses not just the ratio of the discrete Fourier transforms at that frequency, but also a weighted contribution from neighbouring frequencies. This weighting, often called the frequency window, provides some smoothing of the frequency response, and is founded on the principle that the true frequency response is a smooth function of $\omega$. See [28, chap. 6] for more details on this approach. The agreement between the reduced-order models and the spectral estimates is excellent over all frequencies for both the gain and the phase. Although the two models are each approximations of the true system, we derive confidence from the fact that they are in excellent agreement, given that they are found using completely different methods and input-output data sets. (While the ERA uses an impulse as its input (see $§ 2.3$ and Appendix A), the spectral estimate uses zero-mean Gaussian noise as the forcing signal.)

\subsection{Feedback controller design}

Our ultimate aim is to use feedback control to completely eliminate vortex shedding, thus rendering the flow steady. To do so we will employ model-based linear 

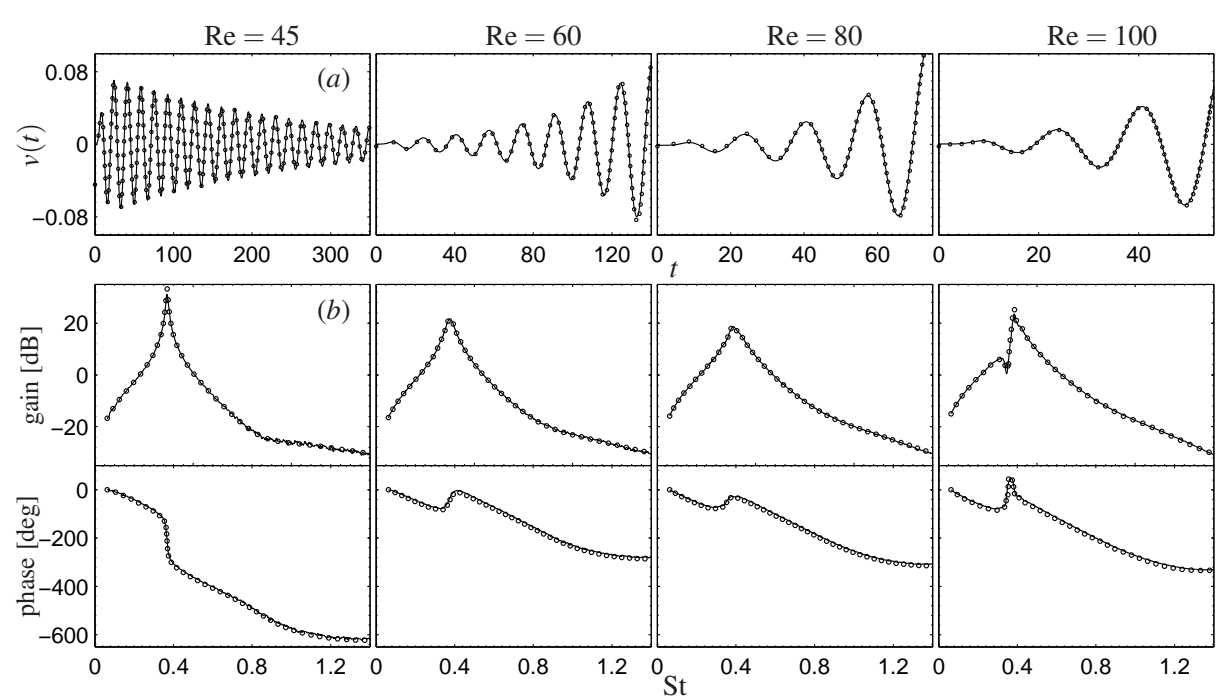

Fig. 2 Validation of the reduced-order models at Reynolds numbers of 45, 60, 80 \& 100: (a) open-loop impulse responses (o) compared to DNS (-); and (b) gain and phase of frequency responses (o) compared to spectral estimates $(-)$. The impulse response for $\mathrm{Re}=45$ is multiplied by 20 so that the same scale can be used.

control. There exist many methods for designing linear controllers based on linear models. These include classical loop-shaping; pole placement methods; Linear Quadratic and Linear Quadratic Gaussian control; and $\mathcal{H}_{2^{-}}$and $\mathcal{H}_{\infty}$-based methods. The method chosen here is $\mathcal{H}_{\infty}$ loop-shaping [30], a modern synthesis method which is well-favoured in the control community. A particular strength is the way in which it consolidates classical control with modern control in a powerful and pleasing way [44]. In order to introduce the method, we must first do two things: move into the frequency domain; and introduce the $\mathcal{H}_{\infty}$-norm. Taking Laplace transforms of (1) and rearranging, we arrive at the transfer function, $P(s)$, defined by $y(s)=P(s) u(s)$, with

$$
P(s)=C(s I-A)^{-1} B+D .
$$

For $p$ inputs and $q$ outputs, $P(s)$ is a matrix of dimension $q \times p$. We can now introduce the $\mathcal{H}_{\infty}$ norm of a transfer function, which is defined for stable systems $\operatorname{as}^{1}$

$$
\|P\|_{\infty}=\max _{\omega} \bar{\sigma}(P(j \omega))
$$

That is, an input that comes arbitrarily close to attaining the $\mathcal{H}_{\infty}$ norm concentrates its energy at the frequency where the gain of the system - as measured by the maximum singular value of its frequency response matrix - is largest. In the time domain this means that

$$
\|P\|_{\infty}=\sup _{u \neq 0} \frac{\|P u\|_{2}}{\|u\|_{2}} .
$$

\footnotetext{
1 More generally, the $\mathcal{H}_{\infty}$ norm is taken as the supremum of the maximum singular value over the open right-half plane. However, provided that $P(s)$ is a rational function of $s$, this supremum is actually attained on the boundary $s=j \omega$ (for possibly infinite $\omega$ ).
} 


\subsection{1 $\mathcal{H}_{\infty}$ loop shaping}

Having moved into the frequency domain and defined the $\mathcal{H}_{\infty}$ norm, we now define the standard feedback arrangement considered in the $\mathcal{H}_{\infty}$ loop-shaping method, together with the quantity $b_{P, C}$ used to characterize it:

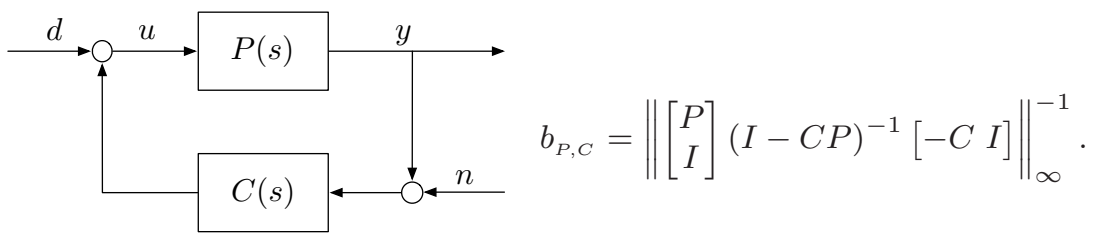

Here $d$ represents disturbances at the input and $n$ represents sensor noise at the output. (The expression given for $b_{P, C}$ is valid only if the feedback interconnection of $P$ and $C$ is stable. Otherwise, $b_{P, C}=0$.)

$b_{P, C}$ is therefore the inverse of the $\mathcal{H}_{\infty}$ norm from disturbances $d$ and noise $n$ to $u$ and $y$ in the standard feedback configuration shown above. The norm cannot be made smaller than 1 which means that, for any $P$ and $C, b_{P, C}$ lies in the range $[0,1]$. A simple interpretation of $b_{P, C}$ is as follows. A large value of $b_{P, C}$ implies that the $\mathcal{H}_{\infty}$ norm of the transfer function matrix in (5) remains small over all frequencies and all directions. The use of $b_{P, C}$ as a performance measure is therefore motivated by the fact that it bounds the gain of all four closed-loop transfer functions at any point in the loop. Furthermore, for single-input-single-output systems, the classical gain and phase margins of the closed-loop can be related to $b_{P, C}$, providing an important and pleasing link to classical control. Glover \& McFarlane [13] show that $b_{P, C}$ can be maximized over all stabilizing $C$ to give

$$
b_{\mathrm{opt}}(P)=\sup _{C} b_{P, C}
$$

and this is the optimization that the $\mathcal{H}_{\infty}$ loop-shaping procedure achieves.

\subsubsection{Control weight $W(s)$}

Like any optimal design method, there are choices to be made about what constitutes optimal. In the present case this amounts to weighting the plant $P$ in the frequency domain using frequency-dependent weights to reflect the control objectives. The controllers to be presented in $\S 3$ have all been designed using the same (non-dimensional) control weight:

$$
W(s)=k \frac{a^{2}}{(s+a)^{2}},
$$

with $a=2 \pi$ and $k$ to be chosen. This weight achieves large gain at low frequencies to reap the benefits of feedback, and small gain at high frequencies to remain robust in the face of modelling uncertainties (which will be greatest at high frequencies). The value of $a$-which determines the bandwidth of the controller, and corresponds to a Strouhal number of St $=a / 2 \pi=2 \pi / 2 \pi=1$ - is chosen to lie well above the frequency of shedding, which occurs at a Strouhal number near 0.12 for all of the Reynolds numbers considered. 
The value of the gain $k$ in (7) is chosen at each Reynolds number. One can think of this as a choice - by the user - of how aggressive the controller should be, with large values of $k$ corresponding to aggressive controllers. More aggressive controllers will typically provide better performance (damping oscillations more rapidly) but poorer robustness (more sensitive to modelling uncertainty and changes in operating conditions). A sensible choice of $k$ is therefore one which provides a good trade-off between performance and robustness. Since $b_{P, C}$ is a good measure of both performance and robustness, a good choice of $k$ is that which maximizes $b_{\text {opt }}$. Appendix $\mathrm{C}$ gives details of the value of $k$ used at each Reynolds number. An important point is that a non-optimal choice of $k$ is to some extent 'optimized away' by the $\mathcal{H}_{\infty}$ loop shaping procedure, and so the value of $b_{\text {opt }}$ achieved is relatively insensitive to the precise choice of $k$. To give an example: For the single-sensor arrangement at a Reynolds number of $60, k=0.31$, for which $b_{\text {opt }}=0.3868$. If we reduce $k$ by a factor of two to $k=0.155$, then $b_{\text {opt }}=0.3586$; if we increase $k$ by a factor of two to $k=0.62$, then $b_{\text {opt }}=0.3611$. Although the final controller is relatively insensitive to the precise choice of $k$, it is important that $k$ is in the vicinity of a sensible value. (This amounts to the user correctly capturing what one wants to achieve with control.) Returning to our example, increasing $k$ by a factor of 10 to $k=3.1$ results in $b_{\text {opt }}=0.2057$, which is a significant reduction. Finally, we note here that we would like to know how the achievable $b_{\text {opt }}$ varies with Reynolds number. It is for this reason that, rather than settle for a $k$ which is merely somewhere within the band of sensible values, the best value of $k$ (as measured by $b_{\text {opt }}$ ) has been used at each Reynolds number. These values are provided in Appendix C.

For the three sensor case, there is one additional control design decision to make: the relative contribution of each sensor signal to the feedback signal. This amounts to choosing a diagonal matrix of dimension three, the diagonal entries of which determine the relative contribution of each sensor. In the simplest case, one can imagine setting this matrix equal to the identity matrix, meaning that each sensor measurement contributes equally. However, the three sensor signals vary somewhat in size, with the sensor furthest downstream providing the largest measurements. In order to make the contribution of each sensor approximately equal, we therefore set the first diagonal entry to 1 , and the second and third entries to numbers less than 1. It has been verified, however, that the same trends in $b_{\text {opt }}$ with Reynolds number (to be presented in $\S 3$ ) are observed for other choices. The precise choice of this weight matrix is given in Appendix C.

\subsubsection{Choice of model order and controller order}

Let us now consider the choice of model order of 100 used for all Reynolds numbers. The controller order needs to be sufficiently low that it can be easily implemented. The controllers generated by $\mathcal{H}_{\infty}$ loop-shaping have the same order as the weighted plant (i.e. $W(s) P(s)$ ). One way to generate low-order controllers is therefore to base them on low-order models ('reduce then design'). We take a different approach. We first design a controller using the $100^{\text {th }}$-order model, and then reduce the order of the controller using balanced truncation ('design then reduce'). The controller order is chosen such that the reduction in $b_{P, C}$ is small. This approach is motivated by the fact that, rather than using the $\mathcal{H}_{\infty}$ norm as in balanced truncation [40] to measure the distance between two systems, it 
is more suitable (for feedback control purposes) to base the controller order on the degradation in $b_{P, C}$ that is causes. (Since the full-order controller for a given plant achieves the optimal $b_{P, C}$, any model reduction must cause a reduction in $\left.b_{P, C}.\right)$ This approach ensures that none of the important dynamics for feedback controller design is 'missed' in the model, and that the performance of the final controller is close enough (as defined by the user) to the best possible. Specifically, the lowest controller order which gives a reduction in $b_{P, C}$ of less than $1 \%$ is used. To give some examples, the order of the single-sensor controllers (including the weight $W(s))$ to be presented in $\S 3.1-3.4$ is 13 for $\operatorname{Re}=45 ; 17$ for $\operatorname{Re}=60 ; 26$ for $\operatorname{Re}=80$; and 32 for $R e=100$. The order of the three-sensor controller (including the weight $W(s))$ for $\operatorname{Re}=100$ to be presented in $\S 3.5$ is 28 . For details of the orders of all designed controllers, see Appendix C.

\section{Model-based feedback control}

We now look at model-based control at Reynolds numbers between 45 and 110 . We first apply control in direct numerical simulations at Reynolds numbers of 45, 60, 80 and 100. We then characterize the performance and robustness of all controllers, which are designed for all Reynolds numbers between 45 and 110, in increments of 5 .

All quantities reported are perturbations away from the steady base flow. For unstable Reynolds numbers, this is the base flow that is attained in closed-loop in the long-time limit. The velocity signals used for feedback are high-pass filtered to ensure that control has no effect on this base flow. One can understand this in the following way: the combination of the controller and high-pass filter together have a DC gain of zero, and therefore control can have no influence at zero frequency, which is by definition the base flow.

The perturbation velocities in the streamwise and transverse directions are $u$ and $v$ respectively. The energy of the perturbations - to be plotted in figures $3-6$ - is then defined as

$$
E(t)=\frac{1}{2} \int_{\mathcal{D}}\left(u^{2}(x, y, t)+v^{2}(x, y, t)\right) \mathrm{d} x \mathrm{~d} y
$$

\subsection{Control at $\mathrm{Re}=45$}

Feedback control in DNS at Re $=45$ is shown in figure 3, which compares the openloop and closed-loop impulse responses. The feedback controller effectively damps out oscillations. This is clear in $(a)$, which shows time traces of the transverse velocity $v$ at the three sensors (whose locations are indicated in $(c, d)$ ). The total perturbation energy with time is shown in $(b)$. The controller reduces the maximum energy over all time to $0.138 / 0.495=28 \%$ of its open-loop value. Finally, vorticity perturbation fields (relative to the base flow) at an instant in time are compared in $(c, d)$. 


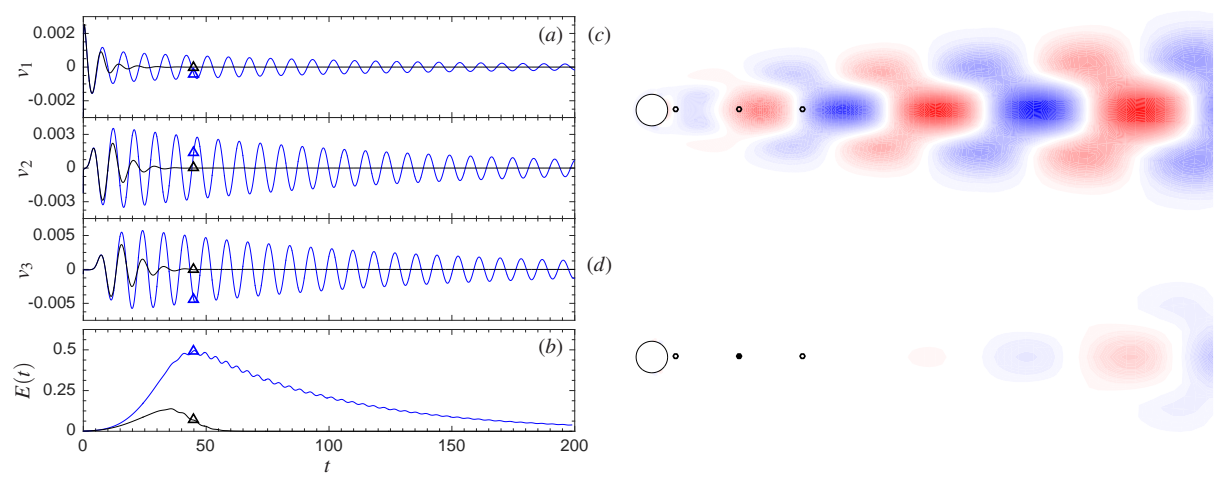

Fig. 3 Control at $\operatorname{Re}=45$ (see supplementary material for an animation): (a) transverse velocity $v$ at the three sensors; (b) total perturbation energy; and $(c, d)$ spatial maps of the vorticity perturbation. Results are shown without control (blue lines and $(c)$ ) and with control (black lines and $(d)$ ). The maximum magnitude of the vorticity perturbation plotted in $(c, d)$ (which use the same colour scale) is $4.96 \times 10^{-3}$. Positive vorticity perturbations are shown in blue, negative perturbations are shown in red, and white corresponds to zero. The instant shown in $(c, d)$ is indicated $(\triangle)$ in $(a, b)$. The location of the feedback sensor $(\bullet)$ and of the remaining sensors (o) is indicated in $(c, d)$.

\subsection{Control at $\operatorname{Re}=60$}

Feedback control in DNS at $\mathrm{Re}=60$ is shown in figure 4. Here the controller designed for $\operatorname{Re}=45, C_{45}$, is compared to the controller designed for this Reynolds number, $C_{60}$. The first thing to note is that $C_{45}$-despite having been designed for a lower Reynolds number at which the wake is stable - still provides closedloop stability when applied at $R e=60$. Furthermore, the performance of $C_{45}$ is very similar to that of $C_{60}$. We see this both in the vorticity perturbation fields in $(c, d)$, which look qualitatively the same, and in the time traces of the velocities in $(a)$, where only minor differences are seen. The best indicator of their similar performance is shown in $(b)$, which compares their total perturbation energy with time. The evolution of the two energies is very similar, with a peak of 1.682 and 1.406 for $C_{45}$ and $C_{60}$, respectively.

\subsection{Control at $\operatorname{Re}=80$}

Feedback control in DNS at Re $=80$ is shown in figure 5. Figure 5 follows the same template as figure 4: The performance of the off-design controller, $C_{60}$, is compared to that of the controller designed for this Reynolds number, $C_{80}$. Once again we see that the off-design controller is sufficiently robust to maintain control when applied at $\operatorname{Re}=80$. This time, however, $C_{80}$ does provide noticeably better performance than $C_{60}$. The velocities at the three sensors in $(a)$ differ significantly, with $C_{80}$ providing the better suppression. We also see in $(b)$ that the total perturbation energy is significantly smaller using $C_{80}$ with a peak value of 12.58 , compared to 35.14 for $C_{60}$. 


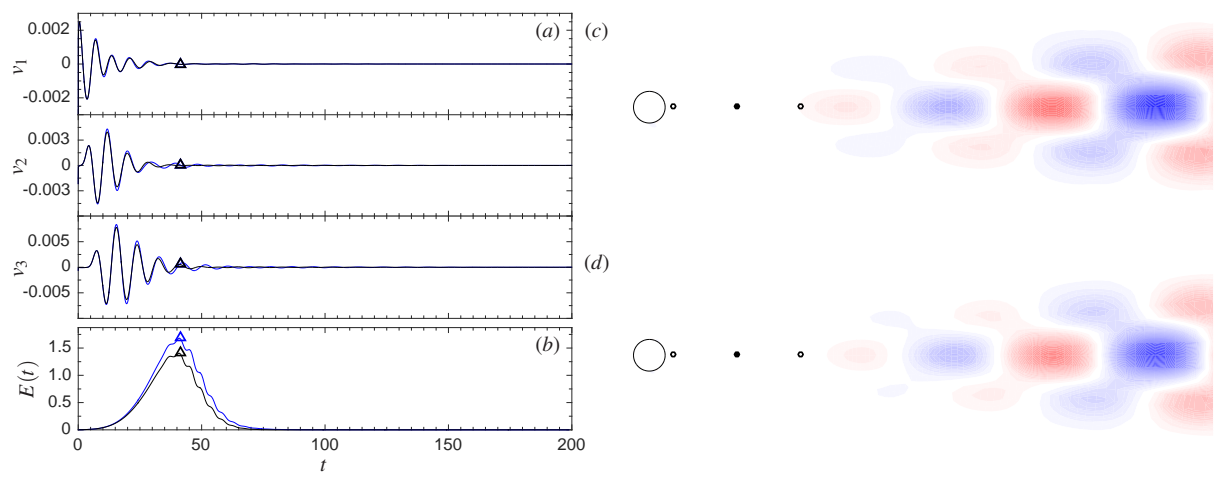

Fig. 4 Control at $R e=60$. (See supplementary material for an animation.) The same template for plotting as figure 3 is used. Results are shown for the $\operatorname{Re}=45$ controller (blue lines and $(c)$ ) and for the $\operatorname{Re}=60$ controller (black lines and $(d)$ ). The maximum magnitude of the vorticity perturbation plotted in $(c, d)$ (which use the same colour scale) is $2.28 \times 10^{-2}$.

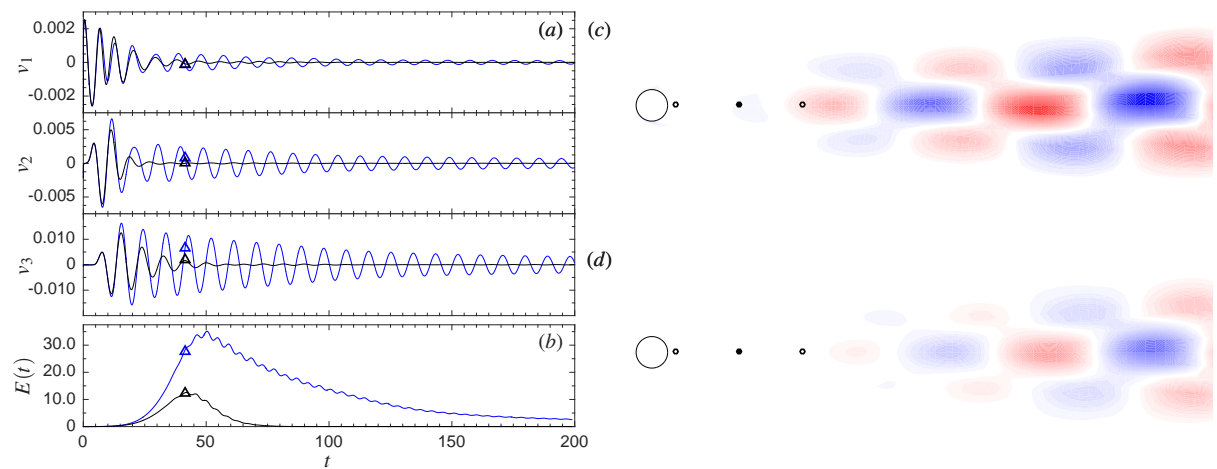

Fig. 5 Control at $R e=80$. (See supplementary material for an animation.) The same template for plotting as figure 3 is used. Results are shown for the Re $=60$ controller (blue lines and $(c)$ ) and for the $\mathrm{Re}=80$ controller (black lines and $(d)$ ). The maximum magnitude of the vorticity perturbation plotted in $(c, d)$ (which use the same colour scale) is $1.10 \times 10^{-1}$.

\subsection{Control at $\operatorname{Re}=100$}

Single-sensor control at $\mathrm{Re}=100$ is significantly more challenging than control at $\operatorname{Re}=45,60$ or 80 . This is revealed in closed-loop simulations in two ways. First, the controller designed for $\mathrm{Re}=80$ in $\S 3.3$ is not successful when applied at $\operatorname{Re}=100$. (See also $\S 3.6$.) Second, a controller designed for $R e=100$, although stabilizing, shows poorer performance than controllers designed for $\operatorname{Re}=45,60$ or 80. This degradation in performance is consistent with the closed-loop results that preceded it: That is, a degradation was already seen between $R e=60$ and $\operatorname{Re}=80$, and this degradation continues to $\operatorname{Re}=100$. This is shown in figure 6 . The degradation in performance is seen most clearly in $(c)$ : although oscillations are small at the sensor location, they are more pronounced further downstream. This is also evident in $(a)$ : the controller does well in reducing oscillations at the feedback sensor location, but oscillations are more severe further downstream. 


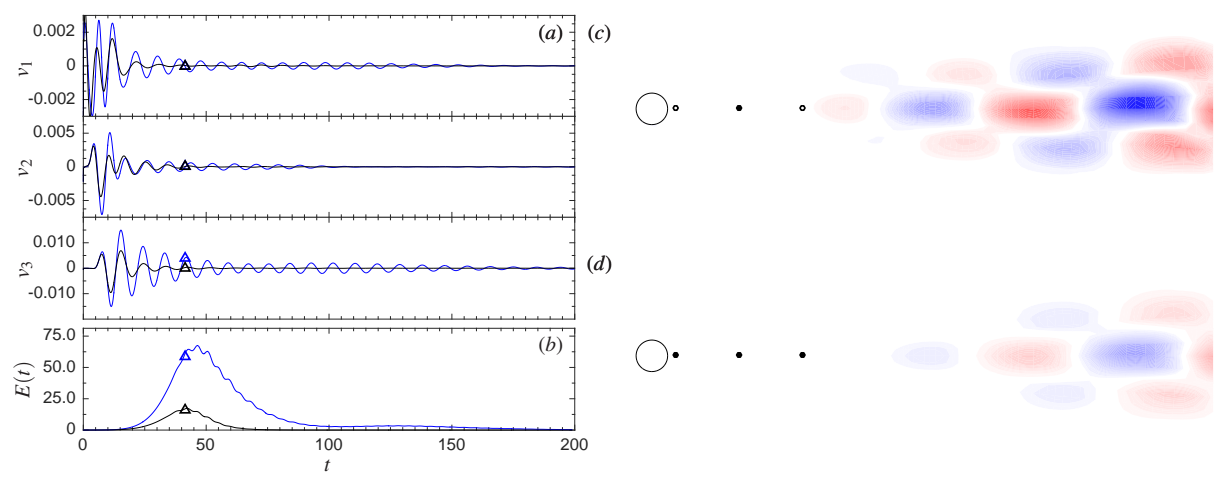

Fig. 6 Control at $R e=100$. (See supplementary material for an animation.) The same template for plotting as figure 3 is used. Results are shown for the single-sensor case (blue lines and $(c)$ ) and for the three-sensor case (black lines and $(d)$ ). The maximum magnitude of the vorticity perturbation plotted in $(c, d)$ (which use the same colour scale) is $1.72 \times 10^{-1}$.

\subsection{Feedback control at $\operatorname{Re}=100$ using all three sensors}

We now consider control at $\mathrm{Re}=100$ when all three sensors are used. The controller is designed and then reduced in the way described in $\S 2.5$ and its order is 28 . Results using all three sensors are shown in figure 6 , where they are compared with the single-sensor case already described in $\S 3.4$. The vorticity perturbation fields in $(c, d)$ clearly show that the three-sensor controller performs significantly better than the single-sensor controller. The velocities at the three sensors in $(a)$ also support this. We also see in $(b)$ that the total perturbation energy is significantly smaller, with a peak value of 17.34 , compared to 67.58 for the single-sensor case. This can be attributed to the controller having access simultaneously to relatively new information from the near wake, and to information from further downstream.

\section{6 * Performance and robustness analysis}

Having looked at the performance of feedback controllers for specific cases, this section gives a broader overview of the performance and robustness of the controllers. We do this by looking at $i$ ) the performance of each controller at its design Reynolds number; and $i$ ) the off-design performance of each controller when applied over a range of off-design Reynolds numbers. We do this both for the singlesensor arrangement considered in $\S 3.1-3.4$, and for the three-sensor arrangement which has so far only been considered for $\mathrm{Re}=100$ in $\S 3.5$.

Before presenting the results we must first make a distinction between the optimal coprime factors stability margin, $b_{\text {opt }}$, defined in (6), and the more general performance measure, $b_{P, C}$, defined in (5). $b_{\text {opt }}$ is the optimal $b_{P, C}$ achieved by a controller at its design Reynolds number. (This is the optimization performed by the $\mathcal{H}_{\infty}$ loop shaping procedure.) When a given controller is applied at an offdesign Reynolds number, we therefore refer to the coprime factor stability margin that it achieves as $b_{P, C}$, rather than $b_{\text {opt }}$, since the controller was not optimized for the Reynolds number under consideration.

The results to be presented are also provided in tables in Appendices D \& E. 


\subsection{1*Single-sensor case}

Figure $7(a)$ shows the $b_{\text {opt }}$ achieved by each controller at its design Reynolds number. This is shown for Reynolds numbers between 45 and 110, in increments of 5 . The plot shows that $b_{\text {opt }}$ reduces monotonically as Reynolds number increases, ranging from a maximum of 0.587 at $R e=45$ to a minimum of 0.146 at $\operatorname{Re}=110$. We will return to this observation in $\S 3.7$.

Let us now consider the performance of each controller at off-design Reynolds numbers. Figure $7(b)$ shows the $b_{P, C}$ achieved across all controller-plant pairs for Reynolds numbers between 45 and 110. The diagonal entries of figure $7(b)$ are thus equivalent to the data plotted in figure $7(a)$ : our interest now lies instead in all off-diagonal points. The colour scale used is such that bluer is better: the bluest controller-plant pairs achieve the highest $b_{P, C}$, and the whitest achieve the lowest. However, recall from $\S 2.5$ that $b_{P, C} \in(0,1]$ if the plant-controller combination is stable, and $b_{P, C}=0$ otherwise (i.e. when the arrangement is unstable). Therefore in order to distinguish between those cases where $b_{P, C}$ is small but nonzero and those points where $b_{P, C}=0$ identically, all plant-controller pairs for which $b_{P, C}=0$ have been coloured in black. Any points which appear white therefore indicate that the closed-loop system is stable, but that the performance measure $b_{P, C}$ is small.

At this point we must bear in mind that $b_{P, C}$ is calculated using a reducedorder model available at each Reynolds number, and therefore one must strictly interpret $b_{P, C}$ as a prediction of the closed-loop stability and performance, rather than as a direct measure of it.

Therefore to supplement and validate the $b_{P, C}$ plots in figure $7(a, b)$, we also plot in parts $(c, d)$ the perturbation energy (defined in Eq. (8)) integrated over time, which we denote by $\mathcal{E}$ :

$$
\mathcal{E}=\int_{T} E(t) \mathrm{d} t
$$

The (non-dimensional) time interval over which this integration is performed is $t \in[0,250]$ for all plant-controller pairs. Since the non-dimensional shedding frequency (i.e. the Strouhal number) remains approximately constant over the range of Reynolds numbers considered, this means that the integral is taken over approximately the same number of cycles in each case. Rather than plot $\mathcal{E}$ directly, we look at its inverse, $\mathcal{E}^{-1}$. We do this because a large value of $\mathcal{E}^{-1}$ suggests good performance, whilst a small value suggests poor performance. This makes it easier to compare it with the values of $b_{P, C}$ in figure $7(a, b)$ (for which a large value is also good). The quantity $\mathcal{E}$ varies by several order of magnitude across all plant-controller pairs. Therefore rather than plot $\mathcal{E}^{-1}$ directly, we instead plot $\log _{10}\left(\mathcal{E}^{-1}\right)=-\log _{10} \mathcal{E}$.

The quantity $\left(-\log _{10} \mathcal{E}\right)$ is plotted in figures $7(c, d)$. These plots follow the same template as parts $(a, b)$, with the design cases (i.e. the diagonal entries) plotted in part $(c)$, and all plant-controller pairs shown as a colour map in part $(d)$. As one might expect, there is broad agreement between the two colour maps: $\mathcal{E}^{-1}$ tends to be large for those plant-controller combinations where $b_{P, C}$ is large, and $\mathcal{E}^{-1}$ is smallest for those plant-controller combinations where $b_{P, C}=0$. (For which - if the predictions of the reduced-order models are correct - the wake should be unstable and therefore develop into a limit-cycle.) 
One lingering question is whether the wake, when simulated in DNS, is indeed stable at those plant-controller combinations where $b_{P, C}>0$, and unstable (resulting in a limit cycle) at those plant-controller combinations where $b_{P, C}=0$. This is difficult to ascertain from figure 7 directly: a large value of $\mathcal{E}$ could occur because the wake is linearly unstable and settles to a limit cycle, or instead because large perturbation energies occur transiently, but ultimately decay. This question has therefore been answered by manually checking the stability of all plant-controller pairs in DNS. The results of this analysis reveal that there is a perfect match between the stability predictions of the reduced-order models (based on $b_{P, C}$ ) and the stability seen in DNS. That is, direct numerical simulations are stable for all plant-controller pairs for which $b_{P, C}>0$, and unstable for all plant-controller pairs for which $b_{P, C}=0$. The fact that the reduced-order models correctly predict the closed-loop stability across all plant-controller pairs is encouraging, and provides further validation of the reduced-order models that have been used.

\subsection{2 *Three-sensor case}

Figure 8 repeats the results of figure 7 , but this time for the three-sensor case described in $\S 3.4$. The overall trends are similar to those seen in figure 7 , with two main differences. First, the controllers are generally more robust, providing stability over a slightly wider range of plant-controller pairs. This manifests itself as fewer black squares (which indicate $b_{P, C}=0$ ) in figure $8(b)$. Second, the quantity $\mathcal{E}^{-1}$ is generally larger $(\mathcal{E}$ smaller) for a given plant-controller pair. (This is most clearly seen by comparing figures $7(d)$ and $8(d)$, which use the same colour scale.) This suggests that perturbations decay more rapidly for this three-sensor case, and therefore that the wake is more highly damped at a given plant-controller combination. Like in figure 7 , the question remains whether the wake, when simulated in DNS, is indeed stable at those plant-controller combinations where $b_{P, C}>0$, and unstable (resulting in a limit cycle) at those plant-controller combinations where $b_{P, C}=0$. Manually checking the stability of all plant-controller pairs in DNS reveals that, again, there is a perfect match between the stability predictions based on the three-sensor reduced-order models with what is actually observed in DNS.

\section{$3.7 *$ Discussion}

It is interesting to note that the $b_{\text {opt }}$ achieved by each controller at its design Reynolds number in figures $7(a)$ and $8(a)$ are strikingly similar for the singlesensor and three-sensor cases. Therefore despite the fact that the three-sensor controllers generally damp oscillations more rapidly - and the fact that they are generally more robust - these improvements in performance and robustness are not reflected directly in the value of $b_{\text {opt }}$ at a given design Reynolds number. It is difficult to attach too much significance to this, since we are ultimately not comparing like-for-like. To compare $b_{\text {opt }}$ for two different single-sensor arrangements - or indeed two different three-sensor arrangements - makes sense, since the number of control loops remains the same. But to compare $b_{\text {opt }}$ across different control arrangements (with different numbers of control loops) makes less sense, so we do not pursue it here. What we certainly can say is that, in both cases, $b_{\text {opt }}$ decreases 

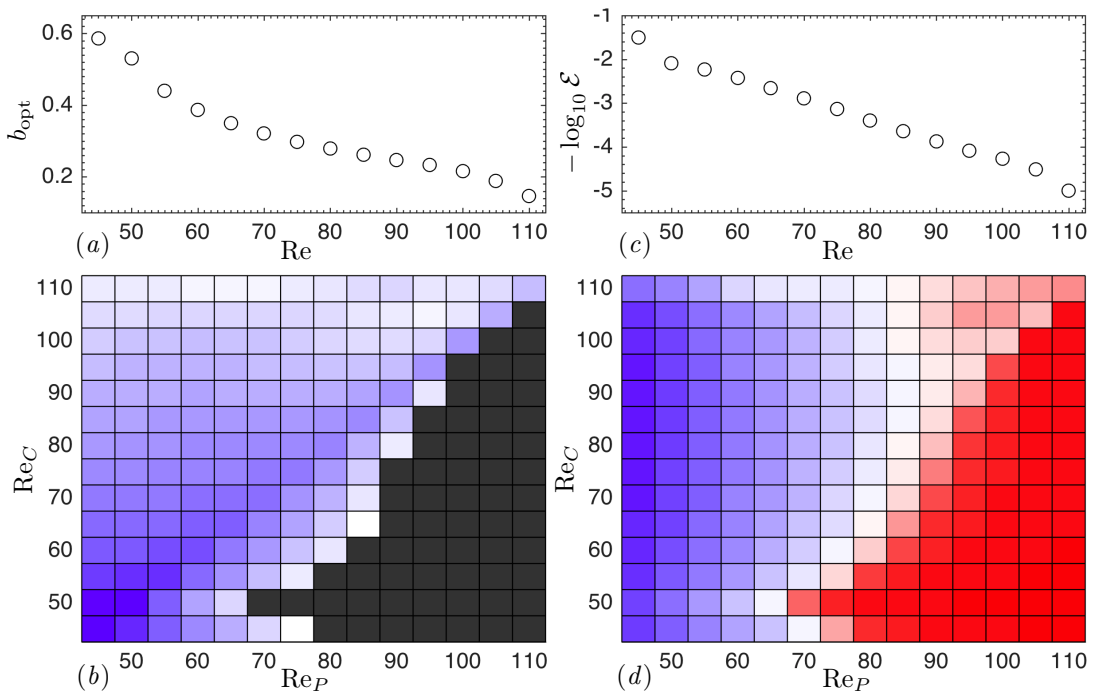

Fig. 7 Performance and robustness of the single-sensor arrangement as measured by $(a, b)$ $b_{P, C}$ and $(c, d)$ the integrated energy $\mathcal{E}$ (see Eq. (9)). ( $a$ ) plots the $b_{\text {opt }}$ achieved by each controller at its design Reynolds number. $(b)$ plots the $b_{P, C}$ achieved across all plant-controller pairs. (The diagonal entries correspond to the data plotted in $(a)$.) Bluer points correspond to larger values of $b_{P, C}$, whiter points to smaller values. Unstable plant-controller combinations (for which $b_{P, C}=0$ ) are shown in black. (c) plots the integrated energy $\left(-\log _{10} \mathcal{E}\right.$ ) for each controller when applied at its design Reynolds number, while $(d)$ plots the same quantity for all plant-controller pairs. Here red represents small values of $\left(-\log _{10} \mathcal{E}\right)$; white represents intermediate values; and blue represents large values. The numerical values for parts $(b)$ and $(d)$ are tabulated in Appendices D and E respectively.
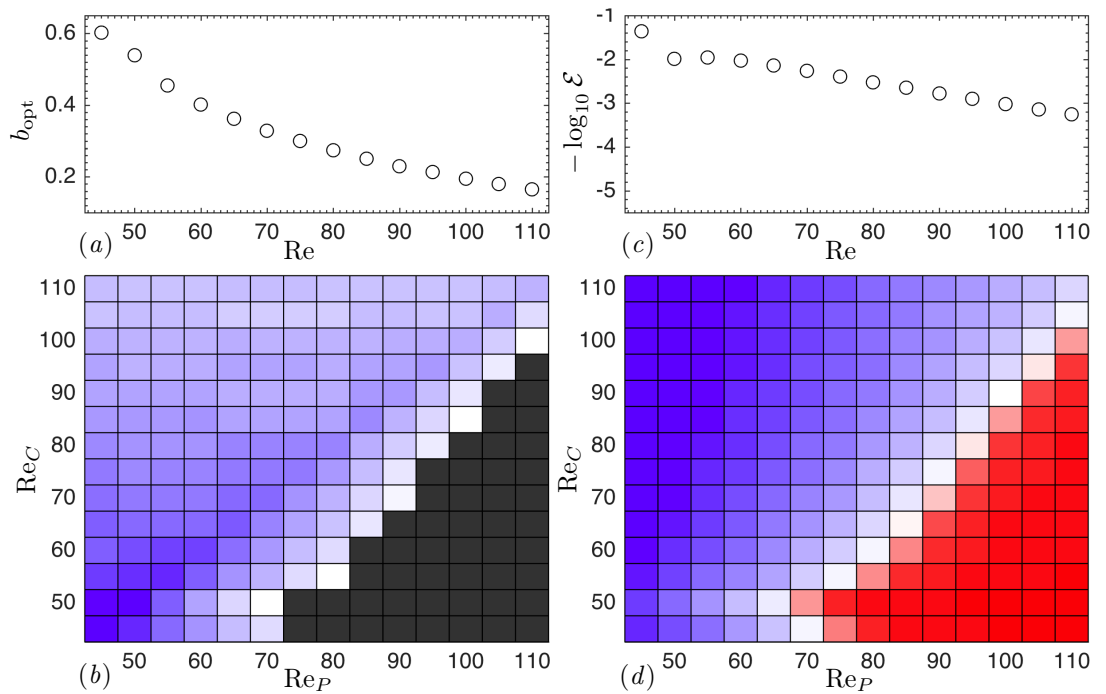

Fig. 8 Performance and robustness of the three-sensor arrangement as measured by $(a, b)$ $b_{P, C}$ and $(c, d)$ the integrated energy $\mathcal{E}$ (see Eq. (9)). Legend same as figure 7 . Parts $(b, d)$ each use the same colour scale as figure $7(b, d)$. The numerical values for parts $(b)$ and $(d)$ are tabulated in Appendices D and E respectively. 
monotonically with Reynolds number, and we explore this now in a little more detail.

$b_{P, C}$ is an excellent measure of robust performance [44]. Following Georgiou \& Smith [11], $b_{\text {opt }}$ (i.e. the achievable $b_{P, C}$ - see Eq. (6)) can also be considered a measure of the ease of control. A value of $b_{\text {opt }}$ near 1 means the plant is 'easy to control', and a value near 0 means the plant is 'hard to control'. The monotonic degradation in $b_{\text {opt }}$ with increasing Reynolds number therefore suggests that feedback control - using the single-sensor or three-sensor arrangements consideredbecomes increasingly difficult as Reynolds number increases. A value of $b_{P, C}$ of approximately 0.3 or above is considered necessary for acceptable robust performance. Using this as a simple metric, we see that acceptable control is not achieved (for either control arrangement) for Reynolds numbers of approximately 80 and above.

The plots of $b_{P, C}$ for the single-sensor arrangement in figure $7(a)$ show that there exists a controller which is stabilizing at all Reynolds numbers considered. Specifically, the controller designed for $\mathrm{Re}=110$ provides closed-loop stability at all Reynolds numbers between 45 and 110 . The smallest $b_{P, C}$ that it achieves is $b_{P, C}=0.0139$, which occurs at a Reynolds number of 65 . Similarly, the plots of $b_{P, C}$ for the three-sensor arrangement in figure $8(a)$ show that, for this arrangement, three of the controllers are stabilizing at all Reynolds numbers. Specifically, the controllers designed for $\operatorname{Re}=100,105$ and 110 are stabilizing at all Reynolds numbers considered. The smallest values of $b_{P, C}$ that the $\mathrm{Re}=100,105$ and 110 controllers achieve are $b_{P, C}=0.0037$ (achieved at $\left.\mathrm{Re}=110\right), b_{P, C}=0.0774$ (achieved at $\operatorname{Re}=110$ ), and $b_{P, C}=0.1209$ (achieved at $R e=90$ ) respectively.

In light of these controllers that are stabilizing across all Reynolds numbers considered, we make two further remarks. First, it would be possible to search for a controller that performs reasonably well (as measured by $b_{P, C}$ ) across all Reynolds numbers. This could be achieved using the extended loop-shaping approach proposed by [44], which sets outs to find

$$
\sup _{C} \inf _{P \in \mathcal{P}} b_{P, C}
$$

That is, to maximize the minimum value of $b_{P, C}$ achieved across the set of plants $\mathcal{P} .^{2}$ This is beyond the scope of the present work, and so we content ourselves here with noting that a stabilizing controller across all Reynolds numbers exists, and that it could be improved upon. Second, it would be interesting to characterize in a precise way the 'distance' between the different plants. For example, taking the $\operatorname{Re}=80$ wake as a nominal model, is the $R e=75$ wake 'closer' to it (with closeness defined in a suitable way), or is the $R e=85$ wake closer? This would reveal potentially interesting trends as Reynolds number varies, and it could be achieved using Vinnicombe's $\nu$-gap metric, $\delta_{\nu}$. This is a suitable measure of the distance between dynamical systems when one's interest lies specifically in their feedback control [44]. Again, this is beyond the scope of the present work, but would be an interesting area to explore.

Finally, it is helpful at this point to link the results presented in figure $8(a)$ back to the 'leapfrogging' approach to system identification described in $\S 2.3$. (Since system identification is performed on the three-sensor arrangement, figure

\footnotetext{
2 Depending on the nature of the set $\mathcal{P}$, this can be a very difficult problem, and so the first step is to embed the set $\mathcal{P}$ inside a set with nicer properties.
} 
$7(a)$ is not relevant here.) The $b_{P, C}$ map shown in figure $8(a)$ reveals precisely which controllers can be used for closed-loop system identification. For example, for the $R e=60$ wake, any of the controllers between $R e=45$ and $R e=110$ could be used to stabilize it, whilst at $R e=100$, any of the controllers between $\operatorname{Re}=85$ and $R e=110$ could be used.

\section{Conclusions}

The problem of completely eliminating vortex shedding using feedback control has been considered in simulations at low Reynolds numbers. Like many of the studies that precede this one, the actuation (blowing and suction) is surface-based, and the feedback signal is provided by velocity measurements in the wake. Model-based feedback control has been employed throughout, which involves two stages. First, a reduced-order model of the wake is required, and this has been provided by the Eigensystem Realization Algorithm (ERA). Second, a feedback control law is designed, and this has been achieved using $\mathcal{H}_{\infty}$ loop-shaping. All reduced-order models have been validated: first in the time domain by comparing their impulse responses to DNS data; and second in the frequency domain by comparing their frequency responses with those given by a spectral method. We can therefore have confidence in their representation of the wake's linear dynamics.

The robustness properties have been investigated by applying each controller (designed for a specific Reynolds number) over the full range of Reynolds numbers considered (i.e. $\mathrm{Re}=45$ to $\mathrm{Re}=110$ in increments of 5 ). It has been observed that, as well as providing closed-loop stability at its design Reynolds number, each controller also maintains stability over a reasonably wide range of off-design Reynolds numbers. Furthermore it has been observed-for both the single-sensor and three-sensor arrangements - that at least one of the controllers is stabilizing over the full range of Reynolds numbers considered.

The optimal normalized coprime factors stability margin, $b_{\mathrm{opt}}$, has been used as a performance measure throughout the study. As well as providing an excellent measure of robust performance, $b_{\text {opt }}$ can also be considered a measure of the ease of control. The monotonic decrease of $b_{\text {opt }}$ with Reynolds number-both for the single-sensor and three-sensor arrangements - therefore suggests that control becomes increasingly challenging as Reynolds number increases. This is not due to any deficiencies in the controller (as was the case in the earliest studies on this topic), but rather is due to the plant itself, and is consistent with previous results in the literature for the Ginzburg-Landau system [25].

We finish by noting that the control performance seen is specific to the control arrangement studied. Despite this, the conclusions are still quite general, since they apply whenever velocity measurements in the wake serve as the feedback signal, which certainly applies to the majority of previous studies. Velocity measurements can only provide local information, and it seems reasonable to ask if measurements with more global attributes might serve better as a feedback signal. Examples might include a single pressure measurement on the cylinder's surface; the distribution of surface pressure; or the lift force experienced by the cylinder. This seems a promising approach, and the subject of future work. 


\section{A *The Eigensystem Realization Algorithm}

This Appendix describes how the Eigensystem Realization Algorithm (ERA) can be used to form balanced reduced-order models from input-output data of high-dimensional systems.

We first move from the continuous time state-model model (1) to its discrete-time equivalent:

$$
\begin{aligned}
x(k+1) & =A x(k)+B u(k) \\
y(k) & =C x(k)+D u(k),
\end{aligned}
$$

where $k=0,1,2, \ldots$ We do this because the direct numerical simulations (and the ERA) are performed in discrete time. The discrete-time state-space matrices in (11) are obviously not the same as the continuous-time matrices defined in (1). In this Appendix, $A, B, C$ and $D$ refer to the discrete-time (rather than continuous-time) state-space matrices.

The impulse function applied to the system (described in $\S 2.3$ ) is defined in discrete time as

$$
u(k)= \begin{cases}u_{\mathrm{o}} & k=0, \\ 0 & k \neq 0,\end{cases}
$$

with $u_{\mathrm{o}}=0.01$, as described in $\S 2.3$.

We now define the $k^{\text {th }}$ Markov parameter, $Y_{k} \in \mathbb{R}^{q \times p}$, for the system (11):

$$
Y_{k} \triangleq C A^{k-1} B \text {. }
$$

For an impulsive input of the form (12), the $k^{\text {th }}$ Markov parameter, $Y_{k}$, is given simply by the output at time step $k, y(k)$ in (11), normalized by the size of the impulse, $u_{\mathrm{o}}$ :

$$
Y_{k}=\frac{y(k)}{u_{\mathrm{o}}}
$$

(For a more general input sequence, an additional step is required to extract the Markov parameters, $Y_{k}$, from the output data, $y(k)$. See [19, App. A] for details.)

The starting point for the ERA is then the generalized $\alpha q \times \beta p$ Hankel matrix composed of the system Markov parameters:

$$
H(k-1)=\left[\begin{array}{cccc}
Y_{k} & Y_{k+1} & \cdots & Y_{k+\beta-1} \\
Y_{k+1} & Y_{k+2} & \cdots & Y_{k+\beta} \\
\vdots & \vdots & \ddots & \vdots \\
Y_{k+\alpha-1} & Y_{k+\alpha} & \cdots & Y_{k+\alpha+\beta-2}
\end{array}\right]
$$

where $\alpha$ and $\beta$ are integers satisfying $\beta p>n$ and $\alpha q \geq n$, which ensures that $H(k-1)$ is of rank $n$. Recall from (1) that $p$ is the number of inputs, $q$ is the number of outputs, and $n$ is the system order.

Setting $k=1$ for the Hankel matrix, substituting in (13) for the Markov parameters, and decomposing into two matrices, we find that

$$
H(0)=\left[\begin{array}{c}
C \\
C A \\
\vdots \\
C A^{\alpha-1}
\end{array}\right]\left[\begin{array}{llll}
B & A B & \cdots & A^{\beta-1} B
\end{array}\right]=\mathcal{P}_{\alpha} \mathcal{Q}_{\beta},
$$

where $\mathcal{P}_{\alpha} \in \mathbb{R}^{\alpha q \times n}$ is called the observability matrix and $\mathcal{Q}_{\beta} \in \mathbb{R}^{n \times \beta p}$ is called the controllability matrix. The concepts of observability and controllability are important for forming reduced-order models. The observability matrix $\mathcal{P}_{\alpha}$ is linked to the measurement of the state: a system is said to be observable if it is possible to recover its state from measurements of its inputs and outputs. The controllability matrix $\mathcal{Q}_{\beta}$ is linked to the manipulation of the state: a system is said to be controllable if any state $x$ can be reached in a finite time when a suitable input is used. Since the Hankel matrix $H(0)$ is composed of the Markov parameters in (15), 
we see from (16) that the Markov parameters are closely related to these observability and controllability matrices. For more details on the notions of observability and controllability, see $[21]$ or [1].

We now look at using the Eigensystem Realization Algorithm to form a reduced-order model of order $n$. The observability and controllability matrices of the reduced-order model will satisfy $\mathcal{P}_{\alpha}^{T} \mathcal{P}_{\alpha}=\mathcal{Q}_{\beta} \mathcal{Q}_{\beta}^{T}$. The reduced-order model is then called balanced, and this means that the input-output dynamics of the system that are important for feedback control are properly accounted for.

Factorizing the Hankel matrix $H(0)$ using the singular value decomposition and truncating to order $n$, we have

$$
H(0)=R \Sigma S^{T} \cong R_{n} \Sigma_{n} S_{n}^{T} .
$$

$R_{n}$ and $S_{n}$ are made up of the first $n$ (orthonormal) columns of $R$ and $S$ respectively. $\Sigma_{n}$ is a rectangular matrix, $\Sigma_{n}=\operatorname{diag}=\left[\begin{array}{llll}\sigma_{1} & \sigma_{2} & \cdots & \sigma_{n}\end{array}\right]$, with $\sigma_{1} \geq \sigma_{2} \geq \cdots \geq \sigma_{n} \geq 0$, the first $n$ singular values of $H(0)$. Examining (16) and (17) as a whole, we can write

$$
H(0)=\mathcal{P}_{\alpha} \mathcal{Q}_{\beta} \cong\left(R_{n} \Sigma_{n}^{1 / 2}\right)\left(\Sigma_{n}^{1 / 2} S_{n}^{T}\right) \text {. }
$$

One possible choice for the decomposition of $H(0)$ is then $\mathcal{P}_{\alpha}=R_{n} \Sigma_{n}^{1 / 2}$ and $\mathcal{Q}_{\beta}=\Sigma_{n}^{1 / 2} S_{n}^{T}$, and this choice makes both $\mathcal{P}_{\alpha}$ and $\mathcal{Q}_{\beta}$ balanced in the sense that

$$
\begin{aligned}
& \mathcal{P}_{\alpha}^{T} \mathcal{P}_{\alpha}=\Sigma_{n}^{1 / 2} R_{n}^{T} R_{n} \Sigma_{n}^{1 / 2}=\Sigma_{n}, \\
& \mathcal{Q}_{\beta} \mathcal{Q}_{\beta}^{T}=\Sigma_{n}^{1 / 2} S_{n}^{T} S_{n} \Sigma_{n}^{1 / 2}=\Sigma_{n} .
\end{aligned}
$$

$\mathcal{P}_{\alpha}^{T} \mathcal{P}_{\alpha}$ and $\mathcal{Q}_{\beta} \mathcal{Q}_{\beta}^{T}$ are called the observability and controllability Gramians of the reducedorder model, and are equal and diagonal by (19).

The $B$ matrix is then given by the first $p$ columns of $\mathcal{Q}_{\beta}$, and the $C$ matrix is given by the first $q$ rows of $\mathcal{P}_{\alpha}$ in (16). To find the $A$ matrix, let $k=2$ in (15) and use (18) to give

$$
H(1)=\mathcal{P}_{\alpha} A \mathcal{Q}_{\beta} \cong\left(R_{n} \Sigma_{n}^{1 / 2}\right) A\left(\Sigma_{n}^{1 / 2} S_{n}^{T}\right),
$$

from which $A$ can be found. For a more detailed treatment of the ERA, see [22] or [21].

The state-space model given by the ERA is balanced: the controllability and observability Gramians are diagonal and equal by (19), and this means that the dynamics of the system are properly taken into account. Furthermore, an upper bound for the error in the reduced-order model can be derived. Let $P_{n}$ denote the transfer function of the reduced-order model of order $n$, and let $P_{r}$ denote the transfer function of the full system. Then the $\mathcal{H}_{\infty}$-norm of the error satisfies

$$
\left\|P_{r}-P_{n}\right\|_{\infty} \leq 2 \sum_{j=n+1}^{r} \sigma_{j},
$$

('twice the sum of the tails'), where $\sigma_{j}$ are the Hankel singular values of $P_{r}$.

Finally, we briefly note a connection to POD here: the modes found by POD (for the specific case of an impulsive input of the form (12)) are the most controllable modes, and the observability of the modes is not taken into consideration. This helps to explain some of the inadequacies of POD for feedback control, since the most controllable modes may be only weakly observable.

\section{B * System identification in closed-loop}

This Appendix describes in more detail the closed-loop approach to system identification.

The ERA is only applicable to stable systems. We can see the problem with applying the ERA to an unstable system by again considering (13):

$$
Y_{k} \triangleq C A^{k-1} B \text {. }
$$

For a stable system subjected to an impulse, the term $A^{k-1}$ becomes progressively smaller, and the Markov parameter (or impulse response) $Y_{k} \simeq 0$ for sufficiently large $k$. For an unstable 
system, however, $A^{k-1}$ becomes progressively larger, and no such approximation can be made. (A similar problem can occur for lightly damped systems, where the $k$ required to satisfy $Y_{k} \simeq 0$ can become prohibitively large.)

The cylinder wake is unstable at all Reynolds numbers considered (with the exception of $\operatorname{Re}=45$, for which the wake is stable). We therefore remedy this problem by applying the ERA to the closed-loop, stable system. (For the $R e=45$ wake, we simply apply standard ERA to the open-loop system.) The closed-loop arrangement on which system identification is performedtogether with the closed-loop transfer function from the input $r$ to the output $y$-is shown below:

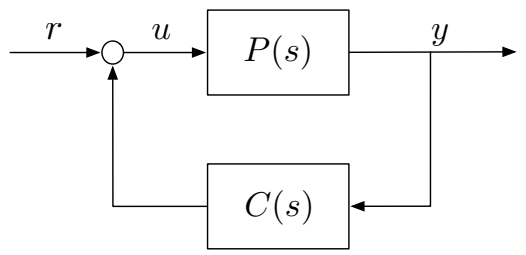

$$
y=(I-P C)^{-1} \operatorname{Pr} .
$$

The closed-loop transfer function from $r$ to $y, P_{\mathrm{CL}}$, is therefore given by

$$
P_{\mathrm{CL}}=(I-P C)^{-1} P .
$$

Applying the ERA to the closed-loop system (i.e. applying the impulse (12) to the signal $r$ rather than $u$ ) provides an estimate of $P_{\mathrm{CL}}$, but what we would like is an estimate of the open-loop system, $P$. Since we know the controller $C$ exactly, we can rearrange (22) to give an expression for $P$ in terms of $P_{\mathrm{CL}}$ and $C$ :

$$
P=\left(I+C P_{\mathrm{CL}}\right)^{-1} P_{\mathrm{CL}} .
$$

The closed-loop system identification is performed for the three-sensor arrangement, which gives an open-loop, reduced-order model $P$ with a single input and three outputs (see figure 1). To form the single-sensor reduced-order model, one then simply picks out the output of interest (velocity sensor 2 in figure 1) from this three-sensor model.

\section{C * Control design}

This Appendix provides additional details relating to controller design. For each Reynolds number, the tables below provide the value of $k$ used in (7); and the final controller order after model reduction (see $\S 2.5 .3$ ). This is done for the single-sensor arrangement in table 1 ; and for the three-sensor arrangement in table 2.

For the three-sensor arrangement, the $3 \times 3$ weight matrix is the same for all Reynolds numbers:

$$
W=\operatorname{diag}\left[\begin{array}{lll}
1 & 0.38 & 0.16
\end{array}\right] \text {. }
$$

Table 1 Single-sensor arrangement: $k$ used in (7) and final controller order at each Reynolds number.

\begin{tabular}{lcccccccccccccc}
\hline Re & 45 & 50 & 55 & 60 & 65 & 70 & 75 & 80 & 85 & 90 & 95 & 100 & 105 & 110 \\
\hline$k$ & 0.32 & 0.15 & 0.24 & 0.31 & 0.35 & 0.39 & 0.42 & 0.44 & 0.47 & 0.51 & 0.56 & 0.63 & 0.70 & 0.61 \\
order & 13 & 10 & 15 & 17 & 19 & 19 & 20 & 26 & 30 & 30 & 30 & 32 & 36 & 42 \\
\hline
\end{tabular}


Table 2 Three-sensor arrangement: $k$ used in (7) and final controller order at each Reynolds number.

\begin{tabular}{lcccccccccccccc}
\hline Re & 45 & 50 & 55 & 60 & 65 & 70 & 75 & 80 & 85 & 90 & 95 & 100 & 105 & 110 \\
\hline$k$ & 0.66 & 0.32 & 0.60 & 0.82 & 0.99 & 1.14 & 1.27 & 1.39 & 1.50 & 1.60 & 1.70 & 1.80 & 1.89 & 1.99 \\
order & 14 & 13 & 17 & 17 & 17 & 17 & 18 & 22 & 27 & 28 & 30 & 28 & 33 & 34 \\
\hline
\end{tabular}

\section{D *Tables of $b_{P, C}$ for all plant-controller pairs}

This Appendix provides tabulated values of all $b_{P, C}$ plotted in figures $7(b)$ and $8(b)$. This is done for the single-sensor arrangement in table 3 ; and for the three-sensor arrangement in table 4 .

Table 3 Single-sensor arrangement: $b_{P, C}$ achieved across all plant-controller pairs.

\begin{tabular}{lcccccccccccccc}
\hline & $P_{45}$ & $P_{50}$ & $P_{55}$ & $P_{60}$ & $P_{65}$ & $P_{70}$ & $P_{75}$ & $P_{80}$ & $P_{85}$ & $P_{90}$ & $P_{95}$ & $P_{100}$ & $P_{105}$ & $P_{110}$ \\
\hline$C_{45}$ & 0.587 & 0.454 & 0.353 & 0.265 & 0.178 & 0.094 & 0.006 & 0 & 0 & 0 & 0 & 0 & 0 & 0 \\
$C_{50}$ & 0.527 & 0.530 & 0.343 & 0.211 & 0.094 & 0 & 0 & 0 & 0 & 0 & 0 & 0 & 0 & 0 \\
$C_{55}$ & 0.433 & 0.437 & 0.440 & 0.335 & 0.235 & 0.141 & 0.045 & 0 & 0 & 0 & 0 & 0 & 0 & 0 \\
$C_{60}$ & 0.373 & 0.377 & 0.382 & 0.387 & 0.302 & 0.221 & 0.137 & 0.049 & 0 & 0 & 0 & 0 & 0 & 0 \\
$C_{65}$ & 0.329 & 0.334 & 0.340 & 0.345 & 0.350 & 0.275 & 0.197 & 0.111 & 0.003 & 0 & 0 & 0 & 0 & 0 \\
$C_{70}$ & 0.291 & 0.297 & 0.303 & 0.309 & 0.315 & 0.321 & 0.248 & 0.166 & 0.059 & 0 & 0 & 0 & 0 & 0 \\
$C_{75}$ & 0.259 & 0.265 & 0.272 & 0.278 & 0.284 & 0.291 & 0.298 & 0.219 & 0.112 & 0 & 0 & 0 & 0 & 0 \\
$C_{80}$ & 0.232 & 0.238 & 0.244 & 0.251 & 0.257 & 0.258 & 0.264 & 0.279 & 0.172 & 0.042 & 0 & 0 & 0 & 0 \\
$C_{85}$ & 0.204 & 0.210 & 0.217 & 0.224 & 0.225 & 0.220 & 0.225 & 0.237 & 0.261 & 0.129 & 0 & 0 & 0 & 0 \\
$C_{90}$ & 0.178 & 0.185 & 0.191 & 0.199 & 0.189 & 0.185 & 0.186 & 0.184 & 0.200 & 0.247 & 0.050 & 0 & 0 & 0 \\
$C_{95}$ & 0.151 & 0.158 & 0.166 & 0.170 & 0.158 & 0.154 & 0.142 & 0.131 & 0.134 & 0.156 & 0.233 & 0 & 0 & 0 \\
$C_{100}$ & 0.119 & 0.127 & 0.134 & 0.141 & 0.131 & 0.124 & 0.107 & 0.088 & 0.078 & 0.072 & 0.099 & 0.216 & 0 & 0 \\
$C_{105}$ & 0.074 & 0.082 & 0.090 & 0.098 & 0.091 & 0.090 & 0.095 & 0.082 & 0.063 & 0.036 & 0.022 & 0.048 & 0.189 & 0 \\
$C_{110}$ & 0.029 & 0.036 & 0.028 & 0.015 & 0.014 & 0.021 & 0.033 & 0.047 & 0.065 & 0.083 & 0.065 & 0.056 & 0.077 & 0.146 \\
\hline
\end{tabular}

Table 4 Three-sensor arrangement: $b_{P, C}$ achieved across all plant-controller pairs.

\begin{tabular}{ccccccccccccccc}
\hline & $P_{45}$ & $P_{50}$ & $P_{55}$ & $P_{60}$ & $P_{65}$ & $P_{70}$ & $P_{75}$ & $P_{80}$ & $P_{85}$ & $P_{90}$ & $P_{95}$ & $P_{100}$ & $P_{105}$ & $P_{110}$ \\
\hline$C_{45}$ & 0.602 & 0.456 & 0.336 & 0.236 & 0.149 & 0.071 & 0 & 0 & 0 & 0 & 0 & 0 & 0 & 0 \\
$C_{50}$ & 0.524 & 0.539 & 0.350 & 0.212 & 0.099 & 0.005 & 0 & 0 & 0 & 0 & 0 & 0 & 0 & 0 \\
$C_{55}$ & 0.423 & 0.454 & 0.455 & 0.348 & 0.250 & 0.161 & 0.079 & 0.001 & 0 & 0 & 0 & 0 & 0 & 0 \\
$C_{60}$ & 0.357 & 0.398 & 0.400 & 0.402 & 0.313 & 0.230 & 0.150 & 0.073 & 0 & 0 & 0 & 0 & 0 & 0 \\
$C_{65}$ & 0.357 & 0.338 & 0.340 & 0.342 & 0.362 & 0.281 & 0.202 & 0.124 & 0.048 & 0 & 0 & 0 & 0 & 0 \\
$C_{70}$ & 0.322 & 0.300 & 0.302 & 0.304 & 0.327 & 0.328 & 0.249 & 0.171 & 0.093 & 0.019 & 0 & 0 & 0 & 0 \\
$C_{75}$ & 0.291 & 0.267 & 0.269 & 0.272 & 0.297 & 0.299 & 0.300 & 0.220 & 0.141 & 0.064 & 0 & 0 & 0 & 0 \\
$C_{80}$ & 0.264 & 0.238 & 0.241 & 0.243 & 0.271 & 0.273 & 0.274 & 0.274 & 0.194 & 0.115 & 0.037 & 0 & 0 & 0 \\
$C_{85}$ & 0.215 & 0.228 & 0.231 & 0.233 & 0.207 & 0.205 & 0.204 & 0.202 & 0.251 & 0.171 & 0.089 & 0.006 & 0 & 0 \\
$C_{90}$ & 0.197 & 0.205 & 0.208 & 0.210 & 0.189 & 0.187 & 0.186 & 0.183 & 0.227 & 0.230 & 0.149 & 0.062 & 0 & 0 \\
$C_{95}$ & 0.203 & 0.176 & 0.179 & 0.181 & 0.201 & 0.198 & 0.195 & 0.192 & 0.201 & 0.203 & 0.213 & 0.126 & 0.036 & 0 \\
$C_{100}$ & 0.186 & 0.160 & 0.163 & 0.166 & 0.178 & 0.175 & 0.173 & 0.169 & 0.174 & 0.177 & 0.185 & 0.194 & 0.105 & 0.004 \\
$C_{105}$ & 0.131 & 0.145 & 0.148 & 0.151 & 0.117 & 0.114 & 0.112 & 0.108 & 0.145 & 0.145 & 0.132 & 0.137 & 0.181 & 0.077 \\
$C_{110}$ & 0.157 & 0.126 & 0.129 & 0.132 & 0.157 & 0.153 & 0.142 & 0.127 & 0.121 & 0.121 & 0.124 & 0.129 & 0.141 & 0.165 \\
\hline
\end{tabular}




\section{$\mathrm{E}{ }^{*}$ Tables of $\mathcal{E}$ for all plant-controller pairs}

This Appendix provides tabulated values of the integrated energy, $\mathcal{E}$ plotted in figures $7(d)$ and $8(d)$. This is done for the single-sensor arrangement in table 5; and for the three-sensor arrangement in table 6.

Table 5 Single-sensor arrangement: $\mathcal{E}$ achieved across all plant-controller pairs.

\begin{tabular}{|c|c|c|c|c|c|c|c|c|c|c|c|c|c|c|}
\hline & $P_{45}$ & 50 & $P_{55}$ & $P_{60}$ & $P_{65}$ & $P_{70}$ & $P_{75}$ & $P_{80}$ & $P_{85}$ & $P_{90}$ & $P_{95}$ & $P_{100}$ & $P_{105}$ & $P_{110}$ \\
\hline$C_{45}$ & $3.17 \mathrm{E} 1$ & $7.66 \mathrm{E} 1$ & $1.87 \mathrm{E} 2$ & $4.62 \mathrm{E} 2$ & $1.21 \mathrm{E} 3$ & $3.86 \mathrm{E} 3$ & $6.32 \mathrm{E} 4$ & $1.46 \mathrm{E} 6$ & $2.46 \mathrm{E} 6$ & $3.15 \mathrm{E} 6$ & $3.61 \mathrm{E} 6$ & $4.03 \mathrm{E} 6$ & $4.40 \mathrm{E} 6$ & $4.50 \mathrm{E} 6$ \\
\hline$C_{50}$ & $4.44 \mathrm{E} 1$ & $1.23 \mathrm{E} 2$ & $3.60 \mathrm{E} 2$ & $1.13 \mathrm{E} 3$ & $4.50 \mathrm{E} 3$ & $3.03 \mathrm{E} 5$ & $1.98 \mathrm{E} 6$ & $2.82 \mathrm{E} 6$ & $3.40 \mathrm{E} 6$ & $3.92 \mathrm{E} 6$ & $4.26 \mathrm{E} 6$ & $4.60 \mathrm{E} 6$ & $5.04 \mathrm{E} 6$ & 8 E6 \\
\hline$C_{55}$ & $2.98 \mathrm{E} 1$ & $7.08 \mathrm{E} 1$ & $1.69 \mathrm{E} 2$ & $4.06 \mathrm{E} 2$ & $1.01 \mathrm{E} 3$ & $2.86 \mathrm{E} 3$ & $1.40 \mathrm{E} 4$ & $8.89 \mathrm{E} 5$ & $1 \mathrm{E} 6$ & $2.93 \mathrm{E} 6$ & $3.54 \mathrm{E} 6$ & $4.04 \mathrm{E} 6$ & E6 & $4.73 \mathrm{E} 6$ \\
\hline$C_{60}$ & $2.40 \mathrm{E} 1$ & $5.36 \mathrm{E} 1$ & $1.19 \mathrm{E} 2$ & $2.64 \mathrm{E} 2$ & $5.95 \mathrm{E} 2$ & $1.41 \mathrm{E} 3$ & $3.83 \mathrm{E} 3$ & $1.79 \mathrm{E} 4$ & $8.68 \mathrm{E} 5$ & $2.08 \mathrm{E} 6$ & $2.88 \mathrm{E} 6$ & $3.43 \mathrm{E} 6$ & 66 & E6 \\
\hline$C_{65}$ & $2.12 \mathrm{E} 1$ & $4.60 \mathrm{E} 1$ & $9.92 \mathrm{E} 1$ & $2.12 \mathrm{E} 2$ & $4.54 \mathrm{E} 2$ & $9.97 \mathrm{E} 2$ & $2.36 \mathrm{E} 3$ & $6.96 \mathrm{E} 3$ & $8.33 \mathrm{E} 4$ & $1.40 \mathrm{E} 6$ & E6 & E6 & 6 & E6 \\
\hline$C_{70}$ & $1.91 \mathrm{E} 1$ & $4.09 \mathrm{E} 1$ & $8.63 \mathrm{E} 1$ & $80 \mathrm{E} 2$ & $3.73 \mathrm{E} 2$ & $\mathrm{E} 2$ & 1.7 & $\mathrm{E} 3$ & $1.63 \mathrm{E} 4$ & 6.5 & E6 & E6 & 3. & $2 \mathrm{E} 6$ \\
\hline$C_{75}$ & $1.78 \mathrm{E} 1$ & $3.78 \mathrm{E} 1$ & $7.89 \mathrm{E} 1$ & $1.62 \mathrm{E} 2$ & $3.29 \mathrm{E} 2$ & $6.64 \mathrm{E} 2$ & $1.37 \mathrm{E} 3$ & $3.05 \mathrm{E} 3$ & $8.67 \mathrm{E} 3$ & 1.90 E5 & $1.56 \mathrm{E} 6$ & $2.49 \mathrm{E} 6$ & $3.19 \mathrm{E} 6$ & $3.80 \mathrm{E} 6$ \\
\hline$C_{80}$ & $1.71 \mathrm{E} 1$ & $3.63 \mathrm{E} 1$ & $7.58 \mathrm{E} 1$ & $1.55 \mathrm{E} 2$ & $3.10 \mathrm{E} 2$ & $6.11 \mathrm{E} 2$ & $1.20 \mathrm{E} 3$ & $2.47 \mathrm{E} 3$ & $5.80 \mathrm{E} 3$ & $2.90 \mathrm{E} 4$ & $1.12 \mathrm{E} 6$ & $2.29 \mathrm{E} 6$ & $3.14 \mathrm{E} 6$ & $3.76 \mathrm{E} 6$ \\
\hline$C_{85}$ & $1.66 \mathrm{E} 1$ & $3.55 \mathrm{E} 1$ & 7.47 E1 & $1.53 \mathrm{E} 2$ & $3.04 \mathrm{E} 2$ & $5.88 \mathrm{E} 2$ & $1.12 \mathrm{E} 3$ & $2.15 \mathrm{E} 3$ & $4.33 \mathrm{E} 3$ & $1.13 \mathrm{E} 4$ & $4.69 \mathrm{E} 5$ & $2.02 \mathrm{E} 6$ & E6 & $.77 \mathrm{E} 6$ \\
\hline$C_{90}$ & $1.66 \mathrm{E} 1$ & $3.62 \mathrm{E} 1$ & $7.72 \mathrm{E} 1$ & $60 \mathrm{E} 2$ & $18 \mathrm{E} 2$ & $6.11 \mathrm{E} 2$ & $1.15 \mathrm{E} 3$ & $2.11 \mathrm{E} 3$ & $3.86 \mathrm{E} 3$ & $7.46 \mathrm{E} 3$ & $3.43 \mathrm{E} 4$ & E6 & 6 & E6 \\
\hline$C_{95}$ & $1.75 \mathrm{E} 1$ & $3.90 \mathrm{E} 1$ & $8.44 \mathrm{E} 1$ & $1.76 \mathrm{E} 2$ & E2 & $6.84 \mathrm{E} 2$ & $1.30 \mathrm{E} 3$ & $2.42 \mathrm{E} 3$ & E3 & E3 & 1.2 & E5 & E6 & E6 \\
\hline$C_{100}$ & $1.99 \mathrm{E} 1$ & $4.43 \mathrm{E} 1$ & $9.55 \mathrm{E} 1$ & $1.99 \mathrm{E} 2$ & $\mathrm{E} 2$ & $7.78 \mathrm{E} 2$ & $1.52 \mathrm{E} 3$ & E3 & E3 & 1.2 & 1.7 & E4 & E6 & 7 E6 \\
\hline$C_{105}$ & $2.73 \mathrm{E} 1$ & $68 \mathrm{E} 1$ & $1.17 \mathrm{E} 2$ & 36 E2 & $\mathrm{E} 2$ & $8.47 \mathrm{E} 2$ & $1.58 \mathrm{E} 3$ & 3.09 & 6.9 & 2.1 & 7. & 6. & \pm 4 & 6 \\
\hline 110 & $1.23 \mathrm{E} 2$ & $1.82 \mathrm{E} 2$ & $4.85 \mathrm{E} 2$ & $67 \mathrm{E} 3$ & $2.82 \mathrm{E} 3$ & $2.99 \mathrm{E} 3$ & $3.47 \mathrm{E} 3$ & $4.52 \mathrm{E} 3$ & $6.73 \mathrm{E} 3$ & $1.17 \mathrm{E} 4$ & $2.35 \mathrm{E} 4$ & $4.78 \mathrm{E} 4$ & $6.77 \mathrm{E} 4$ & $9.94 \mathrm{E} 4$ \\
\hline
\end{tabular}

Table 6 Three-sensor arrangement: $\mathcal{E}$ achieved across all plant-controller pairs.

\begin{tabular}{|c|c|c|c|c|c|c|c|c|c|c|c|c|c|c|}
\hline & $P_{45}$ & $P_{50}$ & $P_{55}$ & $P_{60}$ & $P_{65}$ & $P_{70}$ & $P_{75}$ & $P_{80}$ & $P_{85}$ & $P_{90}$ & $P_{95}$ & $P_{100}$ & $P_{105}$ & $P_{110}$ \\
\hline $\bar{C} C_{45}$ & $2.31 \mathrm{E} 1$ & $5.52 \mathrm{E} 1$ & $1.37 \mathrm{E} 2$ & $3.57 \mathrm{E} 2$ & $1.03 \mathrm{E} 3$ & $3.85 \mathrm{E} 3$ & $1.59 \mathrm{E} 5$ & $1.80 \mathrm{E} 6$ & $2.69 \mathrm{E} 6$ & $3.34 \mathrm{E} 6$ & $3.87 \mathrm{E} 6$ & $4.41 \mathrm{E} 6$ & $6.33 \mathrm{E} 6$ & $6.37 \mathrm{E} 6$ \\
\hline$C_{50}$ & $3.59 \mathrm{E} 1$ & $9.68 \mathrm{E} 1$ & $2.77 \mathrm{E} 2$ & $8.68 \mathrm{E} 2$ & $3.37 \mathrm{E} 3$ & $8.62 \mathrm{E} 4$ & $1.85 \mathrm{E} 6$ & $2.76 \mathrm{E} 6$ & $3.36 \mathrm{E} 6$ & $3.81 \mathrm{E} 6$ & $4.21 \mathrm{E} 6$ & $6.33 \mathrm{E} 6$ & 6 & 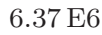 \\
\hline$C_{55}$ & $1.82 \mathrm{E} 1$ & $4.04 \mathrm{E} 1$ & $9.12 \mathrm{E} 1$ & $2.12 \mathrm{E} 2$ & $14 \mathrm{E} 2$ & $1.37 \mathrm{E} 3$ & $4.61 \mathrm{E} 3$ & $1.20 \mathrm{E} 5$ & $1.61 \mathrm{E} 6$ & $2.51 \mathrm{E} 6$ & $3.18 \mathrm{E} 6$ & $3.66 \mathrm{E} 6$ & E6 & E6 \\
\hline$C_{60}$ & E1 & $2.49 \mathrm{E} 1$ & $5.12 \mathrm{E} 1$ & $1.07 \mathrm{E} 2$ & $\mathrm{E} 2$ & $.36 \mathrm{E} 2$ & $1.36 \mathrm{E} 3$ & $4.55 \mathrm{E} 3$ & $1.39 \mathrm{E} 5$ & $1.64 \mathrm{E} 6$ & $2.58 \mathrm{E} 6$ & $3.13 \mathrm{E} 6$ & $3.65 \mathrm{E} 6$ & .05 E6 \\
\hline$C_{65}$ & $16 \mathrm{E} 0$ & $1.81 \mathrm{E} 1$ & $3.49 \mathrm{E} 1$ & $6.87 \mathrm{E} 1$ & 39 E2 & $2.94 \mathrm{E} 2$ & $6.67 \mathrm{E} 2$ & $1.75 \mathrm{E} 3$ & $7.13 \mathrm{E} 3$ & $7.00 \mathrm{E} 5$ & $1.99 \mathrm{E} 6$ & 3 E6 & E6 & 5 E6 \\
\hline$C_{70}$ & $7.74 \mathrm{E} 0$ & $1.41 \mathrm{E} 1$ & $2.60 \mathrm{E} 1$ & $4.85 \mathrm{E} 1$ & $9.27 \mathrm{E} 1$ & $1.84 \mathrm{E} 2$ & $3.85 \mathrm{E} 2$ & $8.89 \mathrm{E} 2$ & $2.56 \mathrm{E} 3$ & $2.17 \mathrm{E} 4$ & $1.37 \mathrm{E} 6$ & $2.28 \mathrm{E} 6$ & $2 \mathrm{E} 6$ & E6 \\
\hline$C_{75}$ & $6.64 \mathrm{E} 0$ & $1.17 \mathrm{E} 1$ & $2.06 \mathrm{E} 1$ & $3.68 \mathrm{E} 1$ & $6.71 \mathrm{E} 1$ & $1.26 \mathrm{E} 2$ & $2.47 \mathrm{E} 2$ & $5.19 \mathrm{E} 2$ & $1.25 \mathrm{E} 3$ & $4.35 \mathrm{E} 3$ & $4.21 \mathrm{E} 5$ & E6 & E6 & E6 \\
\hline$C_{80}$ & $5.87 \mathrm{E} 0$ & 1.0 & $1.71 \mathrm{E} 1$ & 2.9 & E1 & $9.20 \mathrm{E} 1$ & $1.71 \mathrm{E} 2$ & E2 & $\mathrm{E} 2$ & E3 & E3 & E6 & E6 & $1 \mathrm{E} 6$ \\
\hline$C_{85}$ & 5.3 & 8.8 & 1.4 & 2.4 & 4.1 & 7.0 & E2 & $\mathrm{E} 2$ & E2 & E2 & E3 & E4 & $1 \mathrm{E} 6$ & $1 \mathrm{E} 6$ \\
\hline$C_{90}$ & 4.9 & 8.0 & $1.29 \mathrm{E} 1$ & $2.10 \mathrm{E} 1$ & $3.44 \mathrm{E} 1$ & $5.75 \mathrm{E} 1$ & $9.81 \mathrm{E} 1$ & $1.72 \mathrm{E} 2$ & $3.11 \mathrm{E} 2$ & $6.02 \mathrm{E} 2$ & $1.35 \mathrm{E} 3$ & E3 & E5 & $2 \mathrm{E} 6$ \\
\hline$C_{95}$ & $4.66 \mathrm{E} 0$ & $7.41 \mathrm{E} 0$ & $1.17 \mathrm{E} 1$ & $1.86 \mathrm{E} 1$ & $2.98 \mathrm{E} 1$ & $4.85 \mathrm{E} 1$ & $8.05 \mathrm{E} 1$ & $1.36 \mathrm{E} 2$ & $2.35 \mathrm{E} 2$ & $4.19 \mathrm{E} 2$ & $8.04 \mathrm{E} 2$ & $1.88 \mathrm{E} 3$ & $9.65 \mathrm{E} 3$ & $1.30 \mathrm{E} 6$ \\
\hline$C_{100}$ & $4.44 \mathrm{E} 0$ & $6.95 \mathrm{E} 0$ & $1.08 \mathrm{E} 1$ & $1.68 \mathrm{E} 1$ & $2.64 \mathrm{E} 1$ & $4.23 \mathrm{E} 1$ & $6.87 \mathrm{E} 1$ & $1.13 \mathrm{E} 2$ & $1.89 \mathrm{E} 2$ & $3.21 \mathrm{E} 2$ & $5.59 \mathrm{E} 2$ & $1.06 \mathrm{E} 3$ & $2.65 \mathrm{E} 3$ & $6.54 \mathrm{E} 4$ \\
\hline$C_{105}$ & $4.30 \mathrm{E} 0$ & $6.63 \mathrm{E} 0$ & $1.01 \mathrm{E} 1$ & $1.56 \mathrm{E} 1$ & $2.41 \mathrm{E} 1$ & $3.81 \mathrm{E} 1$ & $6.14 \mathrm{E} 1$ & $1.00 \mathrm{E} 2$ & $1.65 \mathrm{E} 2$ & $2.71 \mathrm{E} 2$ & $4.46 \mathrm{E} 2$ & $7.49 \mathrm{E} 2$ & $1.39 \mathrm{E} 3$ & $3.81 \mathrm{E} 3$ \\
\hline$C_{110}$ & $4.16 \mathrm{E} 0$ & $6.34 \mathrm{E} 0$ & $9.59 \mathrm{E} 0$ & $1.45 \mathrm{E} 1$ & $2.23 \mathrm{E} 1$ & $3.50 \mathrm{E} 1$ & $5.62 \mathrm{E} 1$ & $9.18 \mathrm{E} 1$ & $1.51 \mathrm{E} 2$ & $2.46 \mathrm{E} 2$ & $3.94 \mathrm{E} 2$ & $6.20 \mathrm{E} 2$ & $9.93 \mathrm{E} 2$ & $1.82 \mathrm{E} 3$ \\
\hline
\end{tabular}




\section{References}

1. Åström, K.J., Murray, R.M.: Feedback Systems: An Introduction for Scientists and Engineers. Princeton University Press (2008)

2. Bagheri, S., Henningson, D.S., Hoepffner, J., Schmid, P.J.: Input-output analysis and control design applied to a linear model of spatially developing flows. Applied Mech. Rev. 62(2), 020,803 (2009)

3. Berger, E.: Suppression of vortex shedding and turbulence behind oscillating cylinders. Phys. Fluids Suppl. 10, S191-S193 (1967)

4. Bergmann, M., Cordier, L.: Optimal control of the cylinder wake in the laminar regime by trust-region methods and POD reduced-order models. J. Comput. Phys. 227(16), 7813-7840 (2008)

5. Bergmann, M., Cordier, L., Brancher, J.P.: Optimal rotary control of the cylinder wake using proper orthogonal decomposition reduced-order model. Phys. Fluids 17(9), 097,101 (2005)

6. Camarri, S., Iollo, A.: Feedback control of the vortex-shedding instability based on sensitivity analysis. Phys. Fluids 22(9), 094,102 (2010)

7. Cohen, K., Siegel, S., McLaughlin, T., Gillies, E.: Feedback control of a cylinder wake low-dimensional model. AIAA J. 41(7), 1389-1391 (2003)

8. Ffowcs Williams, J.E., Zhao, B.C.: The active control of vortex shedding. J. Fluids Struct. 3(2), 115-122 (1989)

9. Forssell, U., Ljung, L.: Closed-loop identification revisited. Automatica 35(7), 1215-1241 (1999)

10. Fukagata, K., Kasagi, N.: Highly energy-conservative finite difference method for the cylindrical coordinate system. J. Comput Phys. 181(2), 478-498 (2002)

11. Georgiou, T.T., Smith, M.C.: Feedback control and the arrow of time. Int. J. Contr. 83(7), 1325-1338 (2010)

12. Gillies, E.A.: Low-dimensional control of the circular cylinder wake. J. Fluid Mech. 371, 157-178 (1998)

13. Glover, K., McFarlane, D.: Robust stabilization of normalized coprime factor plant descriptions with $\mathcal{H}_{\infty}$-bounded uncertainty. IEEE Trans. Automat. Contr. 34(8), 821-830 (1989)

14. Graham, W.R., Peraire, J., Tang, K.Y.: Optimal control of vortex shedding using loworder models. Part II-model-based control. Internat. J. Numer. Methods Eng. 44(7), 973-990 (1999)

15. Gunzburger, M.D., Lee, H.C.: Feedback control of Karman vortex shedding. J. Applied Mech. 63(3), 828-835 (1996)

16. Gustavsson, I., Ljung, L., Söderström, T.: Identification of processes in closed loopidentifiability and accuracy aspects. Automatica 13(1), 59-75 (1977)

17. He, J.W., Glowinski, R., Metcalfe, R., Nordlander, A., Periaux, J.: Active control and drag optimization for flow past a circular cylinder: I. oscillatory cylinder rotation. J. Comput. Phys. 163(1), 83-117 (2000)

18. Homescu, C., Navon, I.M., Li, Z.: Suppression of vortex shedding for flow around a circular cylinder using optimal control. Int. J. Numer. Meth. Fluids 38(1), 43-69 (2002)

19. Illingworth, S.J., Morgans, A.S., Rowley, C.W.: Feedback control of flow resonances using balanced reduced-order models. J. Sound Vib. 330, 1567-1581 (2011)

20. Illingworth, S.J., Naito, H., Fukagata, K.: Active control of vortex shedding: An explanation of the gain window. Phys. Rev. E 90, 043,014 (2014)

21. Juang, J.N.: Applied System Identification. Prentice Hall (1994)

22. Juang, J.N., Pappa, R.S.: An Eigensystem Realization Algorithm for modal parameter identification and model reduction. J. Guid. Contr. Dyn. 8(5), 620-627 (1985)

23. Keles, R.S.: Active control of transition to turbulence in the wake of a cylinder. Aircraft Design 3(1), 1-15 (2000)

24. Kim, J., Bewley, T.R.: A linear systems approach to flow control. Annu. Rev. Fluid Mech. 39, 383-417 (2007)

25. Lauga, E., Bewley, T.R.: The decay of stabilizability with Reynolds number in a linear model of spatially developing flows. Proc. R. Soc. A 459(2036), 2077-2095 (2003)

26. Li, F., Aubry, N.: Feedback control of a flow past a cylinder via transverse motion. Phys. Fluids 15(8), 2163-2176 (2003)

27. Li, Z., Navon, I.M., Hussaini, M.Y., Dimet, F.X.L.: Optimal control of cylinder wakes via suction and blowing. Comput. \& Fluids 32(2), 149 - 171 (2003) 
28. Ljung, L.: System Identification: Theory for the User, Second Ed., second edn. PrenticeHall (1999)

29. Ma, Z., Ahuja, S., Rowley, C.W.: Reduced order models for control of fluids using the Eigensystem Realization Algorithm. Theor. Comp. Fluid Mech. 25, 233-247 (2009)

30. McFarlane, D.C., Glover, K.: Robust controller design using normalized coprime factor plant descriptions. Springer-Verlag (1990)

31. Min, C., Choi, H.: Suboptimal feedback control of vortex shedding at low Reynolds numbers. J. Fluid Mech. 401, 123-156 (1999)

32. Naito, H., Fukagata, K.: Numerical simulation of flow around a circular cylinder having porous surface. Phys. Fluids 24(11), 117,102 (2012)

33. Park, D.S., Ladd, D.M., Hendricks, E.W.: Feedback control of von Kármán vortex shedding behind a circular cylinder at low Reynolds numbers. Phys. Fluids 6(7), 2390-2405 (1994)

34. Protas, B.: Linear feedback stabilization of laminar vortex shedding based on a point vortex model. Phys. Fluids 16(12), 4473-4488 (2004)

35. Protas, B., Styczek, A.: Optimal rotary control of the cylinder wake in the laminar regime. Phys. Fluids 14(7), 2073-2087 (2002)

36. Roussopoulos, K.: Feedback control of vortex shedding at low Reynolds numbers. J. Fluid Mech. 248(1), 267-296 (1993)

37. Rowley, C.W.: Model reduction for fluids, using balanced proper orthogonal decomposition. Int. J. Bifurc. Chaos 15(3), 997-1013 (2005)

38. Siegel, S., Cohen, K., McLaughlin, T.: Numerical simulations of a feedback-controlled circular cylinder wake. AIAA J. 44(6), 1266-1276 (2006)

39. Singh, S.N., Myatt, J.H., Addington, G.A., Banda, S., Hall, J.K.: Optimal feedback control of vortex shedding using proper orthogonal decomposition models. J. Fluids Engin. 123(3), $612-618(2001)$

40. Skogestad, S., Postlethwaite, I.: Multivariable Feedback Control: Analysis and Design. Wiley (2005)

41. Son, D., Jeon, S., Choi, H.: A proportional-integral-differential control of flow over a circular cylinder. Phil. Trans. R. Soc. A 369(1940), 1540-1555 (2011)

42. Tao, J.S., Huang, X.Y., Chan, W.K.: A flow visualization study on feedback control of vortex shedding from a circular cylinder. J. Fluids Struct. 10(8), 965 - 970 (1996)

43. Van den Hof, P.M.J., Schrama, R.J.P.: Identification and control-closed-loop issues. Automatica 31(12), 1751-1770 (1995)

44. Vinnicombe, G.: Uncertainty and Feedback: $\mathcal{H}_{\infty}$ loop-shaping and the $\nu$-gap metric. Imperial College Press (2000)

45. Weller, J., Camarri, S., Iollo, A.: Feedback control by low-order modelling of the laminar flow past a bluff body. J. Fluid Mech. 634, 405-418 (2009)

46. Williamson, C.H.K.: Oblique and parallel modes of vortex shedding in the wake of a circular cylinder at low Reynolds numbers. J. Fluid Mech. 206, 579-627 (1989)

47. Williamson, C.H.K.: Vortex dynamics in the cylinder wake. Annu. Rev. Fluid Mech. 28, 477-539 (1996) 


\section{University Library}

\section{- M M I N E R VA A gateway to Melbourne's research publications}

Minerva Access is the Institutional Repository of The University of Melbourne

Author/s:

Illingworth, SJ

Title:

Model-based control of vortex shedding at low Reynolds numbers

Date:

2016-10-01

Citation:

Illingworth, S. J. (2016). Model-based control of vortex shedding at low Reynolds numbers. THEORETICAL AND COMPUTATIONAL FLUID DYNAMICS, 30 (5), pp.429-448. https:// doi.org/10.1007/s00162-016-0389-6.

Persistent Link:

http://hdl.handle.net/11343/282898 